\title{
Divergent part of the stress-energy tensor for Maxwell's theory in curved space-time: a systematic derivation
}

\author{
Roberto Niardi $^{1}\left(\mathbb{D}\right.$, Giampiero Esposito $^{1,2, \mathrm{a}}{ }_{\mathbb{D}}$, Francesco Tramontano $^{1,2}$ (D) \\ ${ }^{1}$ Dipartimento di Fisica "Ettore Pancini”, Università degli Studi di Napoli Federico II, Complesso \\ Universitario di Monte S. Angelo, Via Cintia Edificio 6, 80126 Naples, Italy \\ 2 Istituto Nazionale di Fisica Nucleare, Sezione di Napoli, Complesso Universitario di Monte S. Angelo, Via \\ Cintia Edificio 6, 80126 Naples, Italy
}

Received: 31 January 2021 / Accepted: 8 April 2021

(C) The Author(s) 2021

\begin{abstract}
In this paper the Feynman Green function for Maxwell's theory in curved spacetime is studied by using the Fock-Schwinger-DeWitt asymptotic expansion; the pointsplitting method is then applied, since it is a valuable tool for regularizing divergent observables. Among these, the stress-energy tensor is expressed in terms of second covariant derivatives of the Hadamard Green function, which is also closely linked to the effective action; therefore one obtains a series expansion for the stress-energy tensor. Its divergent part can be isolated, and a concise formula is here obtained: by dimensional analysis and combinatorics, there are two kinds of terms: quadratic in curvature tensors (Riemann, Ricci tensors and scalar curvature) and linear in their second covariant derivatives. This formula holds for every space-time metric; it is made even more explicit in the physically relevant particular cases of Ricci-flat and maximally symmetric spaces, and fully evaluated for some examples of physical interest: Kerr and Schwarzschild metrics and de Sitter space-time.
\end{abstract}

\section{Introduction}

The study of quantum gauge field theories and gravitation is a necessity connected to black hole theory and quantum cosmological models, but beyond that it is also a crucial intellectual achievement. On one hand, electroweak and strong interactions are described by Yang-Mills theories, and are satisfactorily set in a quantum framework; on the other hand, although General Relativity is in many ways a gauge theory too [1], it stands apart from the other three forces of nature, and a quantum theory of gravity does not yet exist, at least as a coherent discipline. Nevertheless, it is possible to discuss the influence of the gravitational field on quantum phenomena: one can study the regime for quantum aspects of gravity in which the gravitational field is described as a classical background through Einstein's theory while matter fields are quantized; this is reasonable as long as length and time scales of quantum processes of interest are greater than the Planck values $\left(l_{\text {Planck }} \equiv\left(G \hbar / c^{3}\right)^{1 / 2} \sim 1.616 \times 10^{-33} \mathrm{~cm}\right.$, $t_{\text {Planck }} \equiv\left(G \hbar / c^{5}\right)^{1 / 2} \sim 5.39 \times 10^{-44}$ s). Since Planck length is so small (twenty orders of magnitude below the size of an atomic nucleus), one can hope that such a "semiclassical" approach has some predictive power. Therefore one is naturally led to the subject of quantum

\footnotetext{
a e-mail: Giampiero.Esposito@na.infn.it (corresponding author)
} 
field theory in a curved background spacetime. Its basic physical prediction is that strong gravitational fields can "polarize" the vacuum and, when time-dependent, lead to pair creation; moreover, in a curved space-time, notions of "vacuum" and "particles" need a deeper discussion than in the flat case. These two fundamental results are strongly linked to the most important predictions of the theory, i.e., Hawking and Unruh effects (see Refs. [2-4]): according to the Hawking effect, a classical, spherically symmetric black hole of mass $M$ has the same emission spectrum of a black body having the temperature $T_{\text {Hawking }} \equiv \frac{1}{8 \pi M}$; according to the Unruh effect, from the point of view of an accelerating observer, empty space contains a gas of particles at a temperature proportional to the acceleration. For a detailed treatise on these subjects, see also Refs. [5-7].

In this context, DeWitt's formalism for gauge field theories is a powerful tool (see Refs. [1,8-10]): it provides a framework in which the quantization of fields possessing infinite dimensional invariance groups may be carried out in a manifestly covariant fashion, even in curved space-time; moreover, it makes it possible to classify all gauge theories in a purely geometrical way, i.e., by the algebra which the generators of the gauge group obey; the geometry of such theories is the fundamental reason underlying the emergence of ghost fields in the corresponding quantum theories, too.

For a non-gauge theory, it is well known that the transition amplitude $\langle$ out|in〉 between any two (super)state vectors determined by some unspecified boundary conditions may be expressed as

$$
\begin{aligned}
& \langle\text { out }| \text { in }\rangle=N \int e^{i S[\phi]} \mu[\phi][\mathrm{d} \phi], \\
& {[\mathrm{d} \phi] \equiv \prod_{i} \mathrm{~d} \phi^{i} .}
\end{aligned}
$$

where $N$ is a normalization factor, while $\mu[\phi]$ is a suitable measure functional. ${ }^{1}$ DeWitt regards the set of all fields as an infinite-dimensional manifold, the space of histories, on which an action functional is defined; then gauge transformations are viewed as flows which leave the action functional unchanged; when the flows $Q_{\alpha}$ satisfy the equations of a closed (although infinite-dimensional) Lie group (this is the case for Yang-Mills theories and General Relativity), under the group action the space of histories is separated into orbits; one could say that it is in the space of orbits that the real physics of the system takes place; one can then choose on the manifold a new set of coordinates made of two parts: the first one labelling the orbit, $I^{A}$, and the second one, $K^{\alpha}$, labelling a particular field configuration belonging to the specified orbit; when one writes down the functional integral and reverts to the original coordinates, one finds out that two new terms appear:

$$
\begin{aligned}
\langle\text { out }| \text { in }\rangle & =\int \mu[\phi][\mathrm{d} \phi] \int[\mathrm{d} \chi] \int[\mathrm{d} \psi] e^{i\left(S[\phi]+\Omega+\chi_{\alpha} \mathcal{M}_{\beta}^{\alpha} \psi^{\beta}\right)}, \\
\Omega & \equiv \frac{1}{2} \kappa_{\alpha \beta} K^{\alpha} K^{\beta}, \\
\mathcal{M}_{\beta}^{\alpha} & \equiv K^{\alpha} Q_{\beta},
\end{aligned}
$$

where $\Omega \equiv \frac{1}{2} \kappa_{\alpha \beta} K^{\alpha} K^{\beta}$ is the gauge-breaking (or gauge-averaging) term, while $\chi_{\alpha} \mathcal{M}_{\beta}^{\alpha} \psi^{\beta}$ is the ghost contribution, which involves two ghost fields; they are fermionic for a bosonic

\footnotetext{
1 Although this formula, for a general non-gauge theory, can be derived in a highly formal manner, rigorous results concerning functional integration and path integral can be found in the textbooks [11-13].
} 
theory and bosonic for a fermionic theory. Therefore one refers to

$$
\bar{S} \equiv S[\phi]+\frac{1}{2} \kappa_{\alpha \beta} K^{\alpha} K^{\beta}+\chi_{\alpha} \mathcal{M}_{\beta}^{\alpha} \psi^{\beta}
$$

as the full action functional.

In this paper the Feynman Green function for Maxwell's theory in curved space-time is discussed, following the footsteps of DeWitt, [14] and [15]: both $\zeta$-function regularization method and Fock-Schwinger-DeWitt ansatz are used in order to obtain an asymptotic formula for the Feynman propagator, valid for small values of the geodetic distance; the expansion obtained exhibits the familiar logarithmic singularity which occurs for massive theories in flat space-time and, generally, even for massless theories but in curved space-time. Then the point-splitting method is presented as a valuable tool for regularizing divergent observables such as the stress-energy tensor: one finds out that it can be completely expressed in terms of second derivatives of the Hadamard Green function, which is also closely linked to the effective action. Most of the material in Sects. 2, 3 is quite standard, but nevertheless necessary to obtain a self-contained presentation. In Sect. 4 an original calculation is presented: a short, closed formula for the divergent part of the stress-energy tensor, originating from a careful handling of more than two thousand terms; for this purpose, a program has been written in FORM, which is a symbolic manipulation system whose original author is Jos Vermaseren at NIKHEF (see Refs. [16-18]).

This formula holds for every space-time metric; in Sects. 5 and 6, it is made even more explicit in the physically relevant particular cases of Ricci-flat and maximally symmetric spaces, and fully evaluated for some relevant examples: Kerr and Schwarzschild metrics (which are Ricci-flat) and de Sitter metric (which is maximally symmetric); see Refs. [19,20] for an up-todate review. Concluding remarks are presented in Sect. 7, and relevant details are given in the Appendices.

\section{Quantum Maxwell theory in curved space-time and Feynman propagator}

In this section, the task will be the evaluation of the photon Green's functions in curved space-time. Following DeWitt's approach and using the minimal coupling, the full action functional for the theory is (our spacetime metric has signature $(-,+,+,+)$ )

$$
\bar{S}=\int d^{4} x|\mathrm{~g}|^{1 / 2}\left(-\frac{1}{4} F_{\mu \nu} F^{\mu \nu}-\frac{\left(A_{; \mu}^{\mu}\right)^{2}}{2 \xi}-\frac{\chi \square \psi}{\sqrt{\xi}}\right) .
$$

The first term is the classical action functional for the field $A_{\mu}$, while the second and the third ones are the gauge-averaging and the ghost ones, respectively; moreover, $\xi$ is a real parameter which accounts for the behaviour under rescaling of the gauge-fixing coordinate $K^{\alpha}:$

$$
\begin{aligned}
K^{\alpha} \mapsto & \frac{1}{\sqrt{\xi}} K^{\alpha} \\
\Longrightarrow & \left\{\begin{array}{l}
\Omega \mapsto \frac{1}{\xi} \Omega, \\
M_{\beta}^{\alpha} \mapsto \frac{1}{\sqrt{\xi}} M_{\beta}^{\alpha} .
\end{array}\right.
\end{aligned}
$$


The full action functional (2.1) can be put in the form

$$
\bar{S}=\int d^{4} x|\mathrm{~g}|^{1 / 2}\left(-\frac{1}{2} A_{\mu} P^{\mu \nu}(\xi) A_{\nu}+\frac{1}{\sqrt{\xi}} \chi P_{0} \psi\right),
$$

where

$$
\begin{aligned}
P^{\mu \nu}(\xi) & =-g^{\mu \nu} \square+R^{\mu \nu}+\left(1-\frac{1}{\xi}\right) \nabla^{\mu} \nabla^{v}, \\
P_{0} & =-\square=-g^{\nu \mu} \nabla_{\mu} \nabla_{\nu} .
\end{aligned}
$$

Hence the equation for the photon Green's functions is

$$
|\mathrm{g}|^{1 / 2} P_{\mu}^{\lambda}(\xi) G_{\lambda \nu}^{(\xi)}\left(x, x^{\prime}\right)=g_{\mu \nu}(x) \delta\left(x, x^{\prime}\right) .
$$

In order to study these equations, we introduce two non-physical Hilbert spaces spanned by the basis kets $|x, \mu\rangle,|x\rangle$, respectively; for them, the following orthogonality relations hold:

$$
\begin{aligned}
\left\langle x, \mu \mid x^{\prime}, v\right\rangle & =g_{\mu \nu}(x) \delta\left(x, x^{\prime}\right), \\
\left\langle x \mid x^{\prime}\right\rangle & =\delta\left(x, x^{\prime}\right) .
\end{aligned}
$$

Of course, $|x\rangle$ is the familiar Dirac notation for the eigenfunctionals of the position operator which has continuous spectrum, while the index of both $|x, \mu\rangle$ and the associated "bra" $\langle x, \mu|$ is viewed as that of a covariant vector density of weight $1 / 2$. The "Hamiltonian" operators $H(\xi), H_{0}$ associated with $P^{\mu \nu}(\xi)$ and $P_{0}$, respectively, are defined by

$$
\begin{aligned}
\left\langle x, \mu|H(\xi)| x^{\prime}, v\right\rangle & =P_{\mu}^{\lambda}(\xi)\left\langle x, \lambda \mid x^{\prime}, v\right\rangle, \\
\left\langle x\left|H_{0}\right| x^{\prime}\right\rangle & =P_{0}\left\langle x \mid x^{\prime}\right\rangle .
\end{aligned}
$$

Then the "operator" solution with the Feynman prescription is

$$
|\mathrm{g}|^{1 / 4} G^{(\xi)}|\mathrm{g}|^{1 / 4}=\frac{1}{H(\xi)-i \epsilon}=i \int d \tau e^{-i \tau H(\xi)} .
$$

By taking matrix elements of the previous equation we obtain

$$
\begin{aligned}
|\mathrm{g}(\mathrm{x})|^{1 / 4} G_{\mu \nu}^{(\xi)}\left(x, x^{\prime}\right)\left|\mathrm{g}\left(x^{\prime}\right)\right|^{1 / 4} & =i \int_{0}^{+\infty} d \tau\left\langle x, \mu\left|e^{-i \tau H(\xi)}\right| x^{\prime}, \nu\right\rangle^{(\xi)} \\
& =i \int_{0}^{+\infty} \mathrm{d} \tau\left\langle x, \mu ; \tau \mid x^{\prime}, v ; 0\right\rangle^{(\xi)},
\end{aligned}
$$

where

$$
\left\langle x, \mu ; \tau \mid x^{\prime}, v ; 0\right\rangle \equiv\left\langle x, \mu\left|e^{-i \tau H(\xi)}\right| x^{\prime}, v\right\rangle
$$

Thus the "transition amplitude" $\left\langle x, \mu ; \tau \mid x^{\prime}, v ; 0\right\rangle^{(\xi)}$ satisfies the Schrödinger equation associated to $H(\xi)$ :

$$
i \frac{\partial}{\partial \tau}\left\langle x, \mu ; \tau \mid x^{\prime}, v ; 0\right\rangle^{(\xi)}=\left\langle x, \mu ; \tau|H(\xi)| x^{\prime}, v ; 0\right\rangle^{(\xi)}
$$

with the initial condition

$$
\left\langle x, \mu ; 0 \mid x^{\prime}, v ; 0\right\rangle^{(\xi)}=g_{\mu \nu}(x) \delta\left(x, x^{\prime}\right) .
$$

For arbitrary values of $\xi$, the operator $P^{\mu \nu}(\xi)$, as well as $P_{\mu}^{\lambda} \equiv g_{\rho \mu} P^{\rho \lambda}(\xi)$, is non-minimal, i.e., the wavelike-operator part $-g^{\mu v} \square+R^{\mu \nu}$ is spoiled by $(1-1 / \xi) \nabla^{\mu} \nabla^{v}$. Nevertheless, if one knows $\left\langle x, \mu ; \tau \mid x^{\prime}, v ; 0\right\rangle^{(\xi)}$ at $\xi=1$, one can use this solution, here denoted by 
$\left\langle x, \mu ; \tau \mid x^{\prime}, v ; 0\right\rangle^{(1)}$, to evaluate $\left\langle x, \mu ; \tau \mid x^{\prime}, v ; 0\right\rangle^{(\xi)}$ according to the Endo formula (see Ref. [21])

$$
\left\langle x, \mu ; \tau \mid x^{\prime}, v ; 0\right\rangle^{(\xi)}=\left\langle x, \mu ; \tau \mid x^{\prime}, v ; 0\right\rangle^{(1)}+i \int_{\tau}^{\tau / \xi} d y \nabla_{\mu} \nabla^{\lambda}\left\langle x, \lambda ; y \mid x^{\prime}, v ; 0\right\rangle^{(1)} .
$$

The previous equation plays a key role in evaluating the regularized photon Green function, as we shall see in the following.

Once the transition amplitude is known, equation (2.12) describes the massless limit of the Feynman propagator (for which one would have to add an infinitesimal negative imaginary mass). At this stage, formula (2.12) needs a suitable regularization. Following Endo, we use $\zeta$-function regularization and introduce a regularization parameter $\mu_{A}$ defining (any suffix to denote regularization of the photon Green function is omitted for simplicity of notation):

$$
|\mathrm{g}(\mathrm{x})|^{1 / 4} G_{\mu \nu}^{(\xi)}\left(x, x^{\prime}\right)\left|\mathrm{g}\left(x^{\prime}\right)\right|^{1 / 4} \equiv \lim _{s \rightarrow 0} \frac{\mu_{A}^{2 s} i^{s+1}}{\Gamma(s+1)} \int_{0}^{+\infty} \mathrm{d} \tau \tau^{s}\left\langle x, \mu ; \tau \mid x^{\prime}, \nu ; 0\right\rangle^{(\xi)} .
$$

It should be stressed that the limit $s \rightarrow 0$ should be taken at the very end of all calculations, and cannot be brought within the integral.

The transition amplitude $\left\langle x, \mu ; \tau \mid x^{\prime}, v ; 0\right\rangle^{(1)}$ is known as $\tau \rightarrow 0$ and as

$$
\sigma\left(x, x^{\prime}\right) \rightarrow 0
$$

(where the bi-scalar $\sigma\left(x, x^{\prime}\right)$, which we shall call geodetic interval or world function, is equal to half the square of the distance along the geodesic between $x$ and $x^{\prime}$ ) through its Fock-Schwinger-DeWitt asymptotic expansion (Refs. [1,8,14,22,23])

$$
\begin{aligned}
& \left\langle x, \mu ; \tau\left|x^{\prime}, v ; 0\right|^{(1)} \sim \frac{i}{16 \pi^{2}}|\mathrm{~g}|^{\frac{1}{4}} \sqrt{\Delta}\left|\mathrm{g}^{\prime}\right|^{\frac{1}{4}} e^{\frac{i \sigma}{2 \tau}} \sum_{n=0}^{\infty}(i \tau)^{n-2} b_{n} \mu v^{\prime},\right. \\
& \lim _{x^{\prime} \rightarrow x} b_{0} \mu v^{\prime}=g_{\mu \nu}(x),
\end{aligned}
$$

where $\Delta$ is the Van-Vleck Morette determinant

$$
\Delta \equiv-|\mathrm{g}|^{-1 / 2} \operatorname{det}\left(\sigma_{\mu v^{\prime}}\right)\left|\mathrm{g}^{\prime}\right|^{-1 / 2}
$$

and the coefficient bivectors $b_{n} \mu v^{\prime}$ are evaluated by solving a recursion formula obtained upon insertion of (2.18) into Eq. (2.14); such a recursion formula reads

$$
\begin{aligned}
& \sigma^{; \lambda} b_{0 \mu v^{\prime} ; \lambda}=0, \\
& \sigma^{; \lambda} b_{n+1 \mu v^{\prime} ; \lambda}+(n+1) b_{n+1} \mu v^{\prime} \\
& =\frac{1}{\sqrt{\Delta}}\left(\sqrt{\Delta} b_{n \mu v^{\prime}}\right) ; \lambda \\
& \lambda-R_{\mu}^{\lambda} b_{n} \lambda v^{\prime} .
\end{aligned}
$$

Equations (2.19) and (2.20) are solved by $b_{0 \mu v^{\prime}}=g_{\mu v^{\prime}}$, which is known as the geodetic parallel displacement bi-vector; it is characterized by the following fundamental property: by applying it to a local contravariant vector $V^{\mu^{\prime}}$ at $x^{\prime}$ one obtains the covariant form of the vector which results from displacing $V^{\mu^{\prime}}$ in a parallel fashion along the geodesic from $x^{\prime}$ to $x$ :

$$
g_{\mu v^{\prime}} V^{v^{\prime}}=V_{\mu}
$$


It can be shown (see Ref. [15] for the detailed calculations) that as $x^{\prime}$ approaches $x$ (which implies $\left.\sigma\left(x, x^{\prime}\right) \rightarrow 0\right)$, Eqs. (2.17), (2.18), (2.19) lead to

$$
G_{\mu \nu^{\prime}}^{(\xi)} \sim \frac{i}{16 \pi^{2}} \lim _{s \rightarrow 0} \frac{\mu_{A}^{2 s}}{\Gamma(s+1)} \mathcal{G}_{\mu \nu^{\prime}}^{(\xi)}(s),
$$

where, after having defined

$$
\begin{aligned}
U_{n \mu}^{\lambda}(s ; \xi) & \equiv \frac{2 \delta_{\mu}^{\lambda}}{\sigma\left(x, x^{\prime}\right)}+\frac{\left(\xi^{s+1}-1\right)}{(s+n)(s+1)} \nabla_{\mu} \nabla^{\lambda}, \\
B_{n \lambda v^{\prime}}(s) & \equiv b_{n \lambda \nu^{\prime}} \sqrt{\Delta}\left(x, x^{\prime}\right)\left(\sigma\left(x, x^{\prime}\right) / 2\right)^{s+n},
\end{aligned}
$$

we write

$$
\mathcal{G}_{\mu \nu^{\prime}}^{(\xi)}(s) \equiv \sum_{n=0}^{\infty} \Gamma(1-s-n) U_{n \mu}^{\lambda}(s ; \xi) B_{n \lambda v^{\prime}}(s) .
$$

What is crucial for us is the $s \rightarrow 0$ limit of the sum in the previous equation. Indeed, on studying first, for simplicity, the case when the gauge-field operator reduces to a minimal (wavelike) operator, i.e., at $\xi=1$, one finds

$$
\mathcal{G}_{\mu v^{\prime}}^{(1)}(s)=\frac{2 \sqrt{\Delta}\left(x, x^{\prime}\right)}{\sigma\left(x, x^{\prime}\right)} \sum_{n=0}^{\infty} f_{n \mu v^{\prime}}(s),
$$

having defined

$$
f_{n \mu v^{\prime}}(s) \equiv \Gamma(1-s-n) b_{n \mu v^{\prime}}\left(\sigma\left(x, x^{\prime}\right) / 2\right)^{s+n} .
$$

Since $b_{0 \mu v^{\prime}}=g_{\mu v^{\prime}}$, we therefore find

$$
\mathcal{G}_{\mu \nu^{\prime}}^{(1)}(0)=\frac{2 \sqrt{\Delta}\left(x, x^{\prime}\right)}{\sigma\left(x, x^{\prime}\right)} g_{\mu \nu^{\prime}}+\frac{2 \sqrt{\Delta}\left(x, x^{\prime}\right)}{\sigma\left(x, x^{\prime}\right)} \lim _{s \rightarrow 0} \sum_{n=1}^{\infty} f_{n \mu v^{\prime}}(s),
$$

where the first term on the rhs is precisely the first term in the Hadamard asymptotic expansion at small $\sigma\left(x, x^{\prime}\right)$ (see Ref. [14] ). On the other hand, the Hadamard Green function is, apart from a factor 2, the imaginary part of the Feynman Green function, in agreement with formula (2.22). Eventually, we find therefore, at small $\sigma\left(x, x^{\prime}\right)$,

$$
\begin{aligned}
G_{\mu v^{\prime}}^{(\xi)} \sim & \frac{1}{8 \pi^{2}} \frac{\sqrt{\Delta}\left(x, x^{\prime}\right)}{\sigma\left(x, x^{\prime}\right)+i \epsilon} g_{\mu v^{\prime}}+\frac{i}{16 \pi^{2}} \lim _{s \rightarrow 0}\left[\frac{(\xi-1)}{s(s+1)} \nabla_{\mu} \nabla^{\lambda} B_{0 \lambda v^{\prime}}(s)\right. \\
& \left.+\sum_{n=1}^{\infty} \Gamma(1-s-n) U_{n \mu}{ }^{\lambda}(s ; \xi) B_{n \lambda \nu^{\prime}}(s)\right],
\end{aligned}
$$

i.e., the "flat" Feynman propagator, with the $i \epsilon$ term restored, plus correction resulting from the gauge parameter $(\xi \neq 1$ leading to a non-minimal operator) and from the non-vanishing curvature.

It can be checked (see Ref. [15]) that this infinite sum (2.25) is also able to recover the familiar $\log \sigma\left(x, x^{\prime}\right)$ singularity, which occurs for massive theories in flat space-time and, more generally, even for massless theories (as in our case) but in curved space-time. 


\section{Second derivatives, stress-energy tensor}

In this section it will be shown that, given a field theory (in the present case, quantum Maxwell's theory), there is a close relation between second derivatives of the Hadamard Green's functions, stress-energy tensor and effective action, following the footsteps of Christensen and DeWitt (see Refs. $[9,14]$ ). In a classical field theory, the stress-energy tensor can be obtained as

$$
T^{\mu \nu}=\frac{2}{\sqrt{|\mathrm{g}|}} \frac{\delta S}{\delta g_{\mu \nu}}
$$

For a free theory, this object is quadratic in the fields; when dealing with the associated quantum theory, the expectation value of this observable is, in general, divergent: this happens because the two field operators are taken at the same space-time point. An useful way to regularize the expectation value of the stress-energy tensor is to insert into the formal expression for $T^{\mu \nu}$ not the field operators themselves but operators that have been smeared out by means of a smooth function $s$ of compact support:

$$
\phi_{s}(x) \equiv \int d^{4} y s(x-y) \phi(y) .
$$

The resulting operator does exist and the behaviour of its (finite) expectation value may be studied as the size of the support of $s(x)$ tends to zero. A regularization method equivalent to the one of the smearing method but easier to apply in practice is simply to separate the points at which the two fields in $T^{\mu \nu}$ are taken and then to examine the tensor as the points are brought together again.

For the action functional (2.1), the stress-energy tensor is

$$
T^{\mu \nu}=\frac{2}{\sqrt{|\mathrm{g}|}} \frac{\delta \bar{S}}{\delta g_{\mu \nu}}=T_{\text {Maxwell }}^{\mu \nu}+\frac{1}{\xi} T_{\text {gauge }}^{\mu \nu}+\frac{1}{\sqrt{\xi}} T_{\text {ghost }}^{\mu \nu},
$$

where

$$
\begin{aligned}
T_{\text {Maxwell }}^{\mu \nu} & \equiv \frac{\delta S_{\text {Maxwell }}}{\delta g_{\mu \nu}} \equiv \frac{\delta}{\delta g_{\mu \nu}} \int d^{4} x\left(-\frac{1}{4}|\mathrm{~g}(x)|^{1 / 2} F_{\mu \nu} F^{\mu \nu}\right), \\
T_{\text {gauge }}^{\mu \nu} & \equiv \frac{\delta S_{\text {gauge }}}{\delta g_{\mu \nu}} \equiv \frac{\delta}{\delta g_{\mu \nu}} \int d^{4} x\left(-\frac{1}{2}|g(x)|^{1 / 2}\left(A_{; \mu}^{\mu}\right)^{2}\right), \\
T_{\text {ghost }}^{\mu \nu} & \equiv \frac{\delta S_{\text {ghost }}}{\delta g_{\mu \nu}} \equiv \frac{\delta}{\delta g_{\mu \nu}} \int d^{4} x\left(-|\mathrm{g}(x)|^{1 / 2} \chi \square \psi\right) .
\end{aligned}
$$

The result is

$$
\begin{aligned}
T_{\text {Maxwell }}^{\mu \nu} & =-F_{\rho}^{\mu} F^{\rho v}-\frac{1}{4} F_{\alpha \beta} F^{\alpha \beta} g^{\mu \nu}, \\
T_{\text {gauge }}^{\mu \nu} & =\left(\frac{1}{2}\left(A_{; \alpha}^{\alpha}\right)^{2}+A_{; \alpha \beta}^{\alpha} A^{\beta}\right) g^{\mu \nu}-\left(A_{\alpha}^{; \alpha \mu} A^{v}+A_{\alpha}^{; \alpha \nu} A^{\mu}\right), \\
T_{\text {ghost }}^{\mu \nu} & =\chi_{; \alpha} \psi^{; \alpha} g^{\mu \nu}-\left(\chi^{; \mu} \psi^{; v}+\chi^{; v} \psi^{; \mu}\right) .
\end{aligned}
$$

The detailed calculations are provided in Appendix A. In order to exploit the point split method, we write 


$$
\begin{aligned}
A_{\mu ; \alpha} A_{\rho ; \sigma} & =\frac{1}{2} A_{\mu ; \alpha} A_{\rho ; \sigma}+\frac{1}{2} A_{\rho ; \sigma} A_{\mu ; \alpha} \\
& =\frac{1}{2}\left[A_{\mu ; \alpha}, A_{\rho ; \sigma}\right]_{+} \\
& =\lim _{x^{\prime} \rightarrow x}\left\{\frac{1}{4}\left[A_{\mu ; \alpha}, A_{\rho^{\prime} ; \sigma^{\prime}}\right]_{+}+\frac{1}{4}\left[A_{\mu^{\prime} ; \alpha^{\prime}}, A_{\rho ; \sigma}\right]_{+}\right\}
\end{aligned}
$$

where $[,]_{+}$is the anti-commutator. On the last line, we can pass from classical fields to quantum operators; define the matrix element of an operator $\Theta$ between 〈out, vac| and $\mid$ in, vac $\rangle$ as

$$
\langle\Theta\rangle^{\text {matrix }} \equiv \frac{\langle\text { out, vac }|\Theta| \text { in, vac }\rangle}{\langle\text { out, vac }| \text { in, vac }\rangle} .
$$

Then, evaluating (3.10) between $\langle$ out, vac $|$ and $\mid$ in, vac $\rangle$, dividing by

$$
\langle\text { out, vac|in, vac }\rangle \text {, }
$$

and recalling that, up to a numerical factor, the matrix element of the anticommutator function is the Hadamard Green's function, one obtains ${ }^{2}$

$$
A_{\mu ; \alpha} A_{\rho ; \sigma} \mapsto \lim _{x^{\prime} \rightarrow x}\left\{\frac{1}{4} G_{\mu \rho^{\prime} ; \alpha \sigma^{\prime}}^{(H)}+\frac{1}{4} G_{\rho \mu^{\prime} ; \sigma \alpha^{\prime}}^{(H)}\right\},
$$

where, in order to avoid any confusion with the Feynman Green function in the minimal case $(\xi=1)$, the Hadamard Green function shall be named $G_{\mu v^{\prime}}^{(H)}$. In a similar manner

$$
\begin{aligned}
A_{\mu ; v \rho} A_{\sigma} & =\frac{1}{4}\left[A_{\mu ; v \rho}, A_{\sigma}\right]_{+}+\frac{1}{4}\left[A_{\mu^{\prime} ; v^{\prime} \rho^{\prime}}, A_{\sigma}\right]_{+} \\
& \mapsto \lim _{x^{\prime} \rightarrow x}\left\{\frac{1}{4} G_{\mu \sigma^{\prime} ; v \rho}^{(H)}+\frac{1}{4} G_{\sigma \mu^{\prime} ; v^{\prime} \rho^{\prime}}^{(H)}\right\}
\end{aligned}
$$

and

$$
\chi_{; \mu} \psi_{; v} \mapsto \lim _{x^{\prime} \rightarrow x}\left\{\frac{1}{4} G_{; \mu v^{\prime}}^{(H)}+\frac{1}{4} G_{; \nu \mu^{\prime}}^{(H)}\right\},
$$

where $G^{(H)}\left(x, x^{\prime}\right)$ is the Hadamard Green's function for the ghost fields.

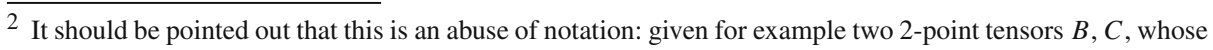
components are $B_{\mu v^{\prime}}, C_{\mu v^{\prime}}$, respectively, one can only sum the components pertaining to the same point and characterized by the same indices: in fact

$$
B_{\mu \nu^{\prime}}+C_{\nu \mu^{\prime}}
$$

does not define a 2-point tensor; therefore, throughout the following,

$$
\lim _{x^{\prime} \rightarrow x}\left\{B_{\mu v^{\prime}}+C_{v \mu^{\prime}}\right\}
$$

stands for

$$
\left(\lim _{x^{\prime} \rightarrow x} B_{\mu v^{\prime}}\right)+\left(\lim _{x^{\prime} \rightarrow x} C_{v \mu^{\prime}}\right)
$$

which is, of course, a tensor at $x$. 
Hence the point-split method yields

$$
\begin{aligned}
\left\langle T^{\alpha \beta}\right\rangle_{\text {Maxwell }}^{\text {matrix }}= & \frac{1}{4} \lim _{x^{\prime} \rightarrow x}\left\{g^{\mu \sigma}\left(g^{\alpha \gamma} g^{\beta \rho}-\frac{1}{4} g^{\alpha \beta} g^{\gamma \rho}\right)\right. \\
& \cdot\left(G_{\sigma \mu^{\prime} ; \rho \gamma^{\prime}}^{(H)}+G_{\mu \sigma^{\prime} ; \gamma \rho^{\prime}}^{(H)}+G_{\rho \gamma^{\prime} ; \sigma \mu^{\prime}}^{(H)}+G_{\gamma \rho^{\prime} ; \mu \sigma^{\prime}}^{(H)}\right. \\
& \left.\left.-G_{\rho \mu^{\prime} ; \sigma \gamma^{\prime}}^{(H)}-G_{\mu \rho^{\prime} ; \gamma \sigma^{\prime}}^{(H)}-G_{\sigma \gamma^{\prime} ; \rho \mu^{\prime}}^{(H)}-G_{\gamma \sigma^{\prime} ; \mu \rho^{\prime}}^{(H)}\right)\right\}, \\
\left\langle T^{\alpha \beta}\right\rangle_{\text {gauge }}^{\text {matrix }}= & \lim _{x^{\prime} \rightarrow x}\left\{-\frac{1}{4} g^{\mu \rho} E^{\alpha \beta \nu \sigma}\left(G_{\rho \sigma^{\prime} ; \mu \nu}^{(H)}+G_{\rho \sigma^{\prime} ; \mu^{\prime} \nu^{\prime}}^{(H)}\right)\right. \\
& \left.+\frac{1}{8} g^{\alpha \beta} g^{\mu \rho} g^{\nu \sigma}\left(G_{\rho \sigma^{\prime} ; \mu \nu^{\prime}}^{(H)}+G_{\sigma \rho^{\prime} ; v \mu^{\prime}}^{(H)}\right)\right\}, \\
\left\langle T^{\alpha \beta}\right\rangle_{\text {ghost }}^{\text {matrix }}= & -\frac{1}{4} \lim _{x^{\prime} \rightarrow x}\left\{E^{\alpha \beta \mu \nu}\left(G_{; \mu \nu^{\prime}}^{(H)}+G_{; \nu \mu^{\prime}}^{(H)}\right)\right\},
\end{aligned}
$$

where the DeWitt supermetric has been introduced:

$$
E^{\mu \nu \rho \tau} \equiv g^{\mu \rho} g^{\nu \tau}+g^{\mu \tau} g^{\nu \rho}-g^{\mu \nu} g^{\rho \tau} .
$$

We should now specify in which order the various operations we rely upon are performed. Indeed, in the evaluation of the Feynman Green function, we first sum over $n$ and then take the $s \rightarrow 0$ limit. Here, we eventually obtain the energy-momentum tensor of the quantum theory according to the point-splitting procedure, with the understanding that the coincidence limit $\lim _{x^{\prime} \rightarrow x}$ is the last operation to be performed.

It is clear from (3.15), (3.16), (3.17) that our analysis of the stress-energy tensor is virtually completed if we can provide a closed expression for the coincidence limit of the second derivatives of the Hadamard Green function. It is easy to see that divergences appear; in the minimal case $(\xi=1)$, on denoting by $\varepsilon$ the regulator that we are employing, the divergent part of $G_{\gamma \beta^{\prime} ; \rho \tau^{\prime}}^{(H)}$ is of the form (see Ref. [15])

$$
\lim _{\varepsilon \rightarrow 0}\left\{\Gamma(\varepsilon)\left(\left[b_{1 \gamma \beta^{\prime} ; \rho \tau^{\prime}}\right]-\frac{1}{6}\left[b_{1 \gamma \beta^{\prime}}\right] R_{\rho \tau}\right)-\frac{1}{2} \Gamma(\varepsilon-1)\left[b_{2 \gamma \beta^{\prime}}\right] g_{\rho \tau}\right\},
$$

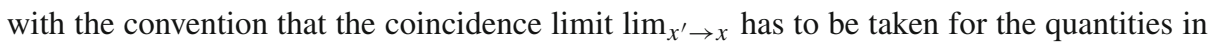
square brackets.

In many applications, we are interested in finding

$$
\left\langle T^{\mu \nu}\right\rangle^{\mathrm{vac}} \equiv\left\langle\text { in, } \operatorname{vac}\left|T^{\mu \nu}\right| \text { in, vac }\right\rangle
$$

the vacuum expectation value of the stress-energy tensor in the vacuum state defined prior to any dynamics in the background gravitational field. This quantity, properly regularized and renormalized, gives us all the information we want about particle production and vacuum polarization. It is the object we choose to use as source in the semiclassical gravitational field equations,

$$
G_{\mu \nu}=\left\langle T_{\mu \nu}\right\rangle^{\mathrm{vac}},
$$

when doing a back-reaction problem. So why are $\left\langle T^{\mu \nu}\right\rangle^{\text {matrix }}$, the Green functions and their divergences interesting? The answer is the following: DeWitt showed (see Ref. [9]) that

$$
\left\langle T^{\mu \nu}\right\rangle^{\text {vac }}=\left\langle T^{\mu \nu}\right\rangle^{\text {matrix }}+\left\langle T^{\mu \nu}\right\rangle^{\text {finite }},
$$

where $\left\langle T^{\mu \nu}\right\rangle^{\text {finite }}$ vanishes when there is no particle annihilation, is always finite, and satisfies the conservation equation $\left\langle T^{\mu \nu}\right\rangle_{; \nu}^{\text {finite }}=0$ (the proof of this beautiful result is described in 
Appendix B). Hence the divergences appearing in $\left\langle T^{\mu \nu}\right\rangle^{\mathrm{vac}}$ and $\left\langle T^{\mu \nu}\right\rangle^{\text {matrix }}$ are identical. Regularization of $\left\langle T^{\mu \nu}\right\rangle^{\text {matrix }}$ yields the regularized form of $\left\langle T^{\mu \nu}\right\rangle^{\mathrm{vac}}$. Regularizing $\left\langle T^{\mu \nu}\right\rangle^{\text {matrix }}$ gives:

$$
\left\langle T^{\mu \nu}\right\rangle^{\text {matrix }}=\left\langle T^{\mu \nu}\right\rangle^{\text {div }}+\left\langle T^{\mu v}\right\rangle^{\text {matrix,ren }},
$$

where $\left\langle T^{\mu \nu}\right\rangle^{\text {div }}$ contains the infinite pieces which we will renormalize away by adding infinite counterterms onto the classical action for the gravitational field and $\left\langle T^{\mu \nu}\right\rangle^{\text {matrix, ren }}$ is the remaining finite physical part of the matrix element. Renormalizing $\left\langle T^{\mu \nu}\right\rangle^{\text {div }}$ away also gives us a renormalized $\left\langle T^{\mu \nu}\right\rangle^{\text {vac }}$

$$
\begin{aligned}
\left\langle T^{\mu \nu}\right\rangle^{\text {vac,ren }} & =\left\langle T^{\mu \nu}\right\rangle^{\text {vac }}-\left\langle T^{\mu \nu}\right\rangle^{\text {div }} \\
& =\left\langle T^{\mu \nu}\right\rangle^{\text {matrix }, \text { ren }}+\left\langle T^{\mu \nu}\right\rangle^{\text {finite }},
\end{aligned}
$$

to be used as the source in (3.21).

Another important fact is the close link between Feynman (and Hadamard) Green's functions and the so-called effective action $W$ : DeWitt showed that

$$
\left\langle T^{\mu \nu}\right\rangle^{\text {matrix }}=2|\mathrm{~g}|^{-1 / 2} \frac{\delta W}{\delta g_{\mu \nu}},
$$

where

$$
W=-i \log \langle\text { out, vac }| \text { in, vac }\rangle \text {. }
$$

Having defined

$$
W=\int d^{4} x L_{\mathrm{eff}}
$$

he also found that

$$
L_{\mathrm{eff}}=\operatorname{Im} \lim _{x^{\prime} \rightarrow x} \operatorname{tr} \frac{\partial}{\partial \sigma}\left(|\mathrm{g}|^{1 / 4}(x) G\left(x, x^{\prime}\right)|\mathrm{g}|^{1 / 4}\left(x^{\prime}\right)\right) .
$$

Thus the renormalized effective Lagrangian is

$$
L_{\text {eff,ren }}=L_{\text {eff }}-L_{\text {div }} \text {, }
$$

where $L_{\text {div }}$ can be evaluated by means of the divergent part of the asymptotic expansion of Feynman and Hadamard Green's functions.

Interestingly, the divergent part of the one-loop effective action for the quantum version of Einstein gravity has been recently discussed in Ref. [24] in relation to renormalization group equations for the Newton constant and the cosmological constant; the reader may find there an up-todate discussion of the concepts just introduced in our section.

\section{Divergent part of $\left\langle T^{\mu v}\right\rangle$}

In this section we will show the results of the calculation pertaining to the divergent part of the coincidence limit of the Hadamard Green function and their application to the evaluation of the divergent part of $\left\langle T^{\mu v}\right\rangle$; the divergent part of the Hadamard Green function is (see (3.19))

$$
\left[G_{\gamma \beta^{\prime} ; \rho \tau^{\prime}}^{(H)}\right]^{\operatorname{div}}=\lim _{\varepsilon \rightarrow 0}\left\{\Gamma(\varepsilon)\left(\left[b_{1 \gamma \beta^{\prime} ; \rho \tau^{\prime}}\right]-\frac{1}{6}\left[b_{1 \gamma \beta^{\prime}}\right] R_{\rho \tau}\right)-\frac{1}{2} \Gamma(\varepsilon-1)\left[b_{2 \gamma \beta^{\prime}}\right] g_{\rho \tau}\right\} .
$$


Thus, upon using

$$
\Gamma(\varepsilon-k)=\frac{1}{\varepsilon} \frac{(-1)^{k}}{k !}+O(1), \quad \text { for } \quad k=0,1,2, \ldots \Longrightarrow\left\{\begin{array}{l}
\Gamma(\varepsilon)=\frac{1}{\varepsilon}+O(1), \\
\Gamma(\varepsilon-1)=-\frac{1}{\varepsilon}+O(1)
\end{array}\right.
$$

together with coincidence limits for the coefficient bivectors and for the remaining geometrical quantities which appear in the Fock-Schwinger-DeWitt asymptotic expansion (most of them are carefully derived in Appendices C, D) one obtains

$$
\begin{aligned}
\varepsilon\left[G_{\gamma \beta^{\prime} ; \rho \tau^{\prime}}^{(H)}\right]^{\mathrm{div}}= & g_{\beta \gamma} g_{\rho \tau}\left(-\frac{1}{360} R_{\alpha_{1} \alpha_{2}} R^{\alpha_{1} \alpha_{2}}+\frac{1}{144} R^{2}\right. \\
& \left.+\frac{1}{360} R_{\alpha_{1} \alpha_{2} \alpha_{3} \alpha_{4}} R^{\alpha_{1} \alpha_{2} \alpha_{3} \alpha_{4}}+\frac{1}{60} \square R\right) \\
& +g_{\beta \gamma}\left(-\frac{1}{135} R_{\alpha_{1} \alpha_{2} \alpha_{3} \rho} R^{\alpha_{1} \alpha_{2} \alpha_{3}}-\frac{1}{135} R_{\alpha_{1} \alpha_{2} \alpha_{3} \rho} R^{\alpha_{1} \alpha_{3} \alpha_{2}}{ }_{\tau}\right. \\
& +\frac{1}{45} R_{\alpha_{1} \rho} R_{\tau}^{\alpha_{1}}+\frac{1}{40} R_{; \rho \tau}-\frac{1}{60} \square R_{\rho \tau}-\frac{1}{36} R R_{\rho \tau} \\
& \left.-\frac{1}{90} R^{\alpha_{1} \alpha_{2}} R_{\alpha_{2} \rho \alpha_{1} \tau}+\frac{1}{20} R_{\rho}^{\alpha_{1}}{ }_{; \alpha_{1} \tau}-\frac{1}{30} R_{\tau}^{\alpha_{1}}{ }_{; \alpha_{1} \rho}\right) \\
& +g_{\rho \tau}\left(\frac{1}{4} R_{\alpha_{1} \beta} R_{\gamma}^{\alpha_{1}}-\frac{1}{12} \square R_{\beta \gamma}-\frac{1}{12} R R_{\beta \gamma}\right. \\
& \left.-\frac{1}{24} R_{\alpha_{1} \alpha_{2} \alpha_{3} \beta} R^{\alpha_{1} \alpha_{2} \alpha_{3}}{ }_{\gamma}\right)+\frac{1}{6} R_{\beta \gamma} R_{\rho \tau} \\
& +\frac{1}{12} R^{\alpha_{1}}{ }_{\gamma \alpha_{2} \tau} R_{\rho \alpha_{1} \beta}^{\alpha_{2}}+\frac{1}{12} R^{\alpha_{1}}{ }_{\gamma \alpha_{2} \rho} R^{\alpha_{2}}{ }_{\tau \alpha_{1} \beta} \\
& +\frac{1}{12} R_{\rho \beta \gamma ; \alpha_{1} \tau}^{\alpha_{1}}-\frac{1}{12} R^{\alpha_{1}}{ }_{\tau \beta \gamma ; \alpha_{1} \rho}+\frac{1}{3} R_{\beta}^{\alpha_{1}} R_{\alpha_{1} \gamma \rho \tau} \\
& \left.-\frac{1}{6} R_{\gamma}^{\alpha_{1}} R_{\alpha_{1} \beta \rho \tau}-\frac{1}{6} R_{\beta \gamma ; \rho \tau}-\frac{1}{12} R R_{\beta \gamma \rho \tau}\right] .
\end{aligned}
$$

In order to obtain the divergent part of $\left\langle T^{\mu \nu}\right\rangle$, one also needs

1. The so-called quantum Ward identities: they constitute a relation between gauge-field Green's functions and ghost Green's function. In our case, they read

$$
\begin{aligned}
& -G_{; \mu}^{(H)}=G_{\mu v^{\prime}}^{(H)} v^{\prime}, \\
& -G_{; v^{\prime}}^{(H)}=G_{\mu v^{\prime}}^{(H)}{ }^{\mu} .
\end{aligned}
$$

Therefore, by taking another covariant derivative, one obtains

$$
\begin{aligned}
-G_{; \mu \rho^{\prime}}^{(H)} & =G_{\mu v^{\prime} ;}^{(H)} \rho^{\prime}, \\
-G_{; v^{\prime} \rho}^{(H)} & =G_{\mu v^{\prime} ;}^{(H)}{ }^{\mu} .
\end{aligned}
$$

2. An expression for the divergent part of the coincidence limit of $G_{\rho \sigma^{\prime} ; \mu \nu}^{(H)}$ and $G_{\rho \sigma^{\prime} ; \mu^{\prime} \nu^{\prime}}^{(H)}$ in terms of $G_{\rho \sigma^{\prime} ; \mu \nu^{\prime}}^{(H)}$. They are easily obtained by using the parallel displacement matrix:

$$
\begin{aligned}
{\left[G_{\rho \sigma^{\prime} ; \mu \nu}^{(H)}\right] } & =\left[G_{\rho \sigma^{\prime} ; \mu \beta^{\prime}}^{(H)} g^{\beta^{\prime}}\right] \\
& =\left[G_{\rho \sigma^{\prime} ; \mu \beta^{\prime}}^{(H)}\right]\left[g^{\beta^{\prime}}\right] \\
& =\left[G_{\rho \sigma^{\prime} ; \mu \beta^{\prime}}^{(H)}\right] \delta_{v}^{\beta} \\
& =\left[G_{\rho \sigma^{\prime} ; \mu v^{\prime}}^{(H)}\right]
\end{aligned}
$$




$$
\begin{aligned}
{\left[G_{\rho \sigma^{\prime} ; \mu^{\prime} v^{\prime}}^{(H)}\right] } & =\left[G_{\rho \sigma^{\prime} ; \beta \nu^{\prime}}^{(H)} g_{\mu^{\prime}}^{\beta}\right] \\
& =\left[G_{\rho \sigma^{\prime} ; \beta v^{\prime}}^{(H)}\right]\left[g_{\mu^{\prime}}^{\beta}\right] \\
& =\left[G_{\rho \sigma^{\prime} ; \beta v^{\prime}}^{(H)}\right] \delta_{\mu}^{\beta} \\
& =\left[G_{\rho \sigma^{\prime} ; \mu v^{\prime}}^{(H)}\right] .
\end{aligned}
$$

The final result is therefore

$$
\begin{aligned}
\varepsilon\left\langle T^{\mu \nu}\right\rangle^{\mathrm{div}}= & g^{\mu \nu}\left(-\frac{23}{180} R_{\alpha \beta} R^{\alpha \beta}+\frac{1}{144} R^{2}-\frac{1}{24} R_{\alpha \beta \gamma \delta} R^{\alpha \gamma \beta \delta}\right. \\
& \left.-\frac{7}{180} R_{\alpha \beta \gamma \delta} R^{\alpha \beta \gamma \delta}+\frac{7}{120} \square R\right) \\
& +\frac{17}{180} R_{\alpha \beta} R^{\alpha \mu \nu \beta}+\frac{127}{1080} R^{\mu \alpha \beta \gamma} R_{\alpha \beta \gamma}^{\nu} \\
& -\frac{49}{540} R^{\mu \alpha \beta \gamma} R_{\beta \alpha \gamma}^{v}+\frac{19}{180} R_{\alpha}^{\mu} R^{\alpha \nu} \\
& +\frac{1}{20}\left(R^{\mu \alpha}{ }^{\nu}{ }^{\nu}+R_{; \alpha}^{\nu \alpha}\right) \\
& +\frac{1}{24}\left(R^{\alpha \mu \nu} ; \alpha+R_{; \alpha}^{\alpha \nu \mu}\right) \\
& \left.-\frac{7}{120} R^{; \mu \nu}+\frac{1}{18} R R^{\mu \nu}-\frac{11}{60} \square R^{\mu \nu}\right] .
\end{aligned}
$$

Although the expressions for the divergent part of $\left[G_{\gamma \beta^{\prime} ; \rho \tau^{\prime}}^{(H)}\right]$ and $\left\langle T^{\mu \nu}\right\rangle^{\text {div }}$ may seem cumbersome, there is a systematic way to deal with them. Indeed, a more careful inspection shows that they result from a very large number of terms: by looking at the coincidence limits in Appendices C, D, it is easy to see that each divergent part of $\left[G_{\gamma \beta^{\prime} ; \rho \tau^{\prime}}^{(H)}\right]$ is obtained from the sum of eighty contractions variously involving the metric tensor, the Ricci and Riemann tensor, and that $\left\langle T^{\mu \nu}\right\rangle^{\text {div }}$ is obtained by summing thirty of these objects; therefore expression (4.10) for $\left\langle T^{\mu \nu}\right\rangle^{\text {div }}$ results from more than two thousand terms. For this purpose, a program has been written in FORM. In the following two sections, Eq. (4.10) will be made more explicit in some physically relevant particular cases.

\section{Ricci-flat space-times}

In light of their role in General Relativity, Ricci-flat metric tensors are very relevant, since they solve the Einstein vacuum equations. Whenever one considers a Ricci-flat metric, only four terms are left in the sum Eq. (4.10), i.e., the ones which only involve the Riemann tensor and its derivatives:

$$
\begin{aligned}
\left.\varepsilon\left\langle T^{\mu \nu}\right\rangle^{\text {div }}\right|_{\text {Ricci-flat }}= & {\left[g^{\mu \nu}\left(-\frac{1}{24} R_{\alpha \beta \gamma \delta} R^{\alpha \gamma \beta \delta}-\frac{7}{180} R_{\alpha \beta \gamma \delta} R^{\alpha \beta \gamma \delta}\right)\right.} \\
& \left.+\frac{127}{1080} R^{\mu \alpha \beta \gamma} R_{\alpha \beta \gamma}^{v}-\frac{49}{540} R^{\mu \alpha \beta \gamma} R_{\beta \alpha \gamma}^{v}\right] .
\end{aligned}
$$

An important example of vacuum solution is the Kerr metric, which describes the exterior of a rotating, stationary, axially symmetric star; it depends on two parameters: the Schwarzschild radius $r_{s} \equiv \frac{2 G M}{c^{2}}$ and the angular momentum $J$, through $a \equiv \frac{J}{M c}$; in a spherical coordinate 
system $(t, r, \theta, \phi)$, the metric components $g_{\mu \nu}$ form the matrix

$$
g_{\mu \nu}=\left(\begin{array}{cccc}
g_{00} & 0 & 0 & g_{03} \\
0 & g_{11} & 0 & 0 \\
0 & 0 & g_{22} & 0 \\
g_{30} & 0 & 0 & g_{33}
\end{array}\right)
$$

where

$$
\begin{aligned}
& g_{00}=\frac{r r_{s}}{r^{2}+a^{2} \cos ^{2}(\theta)}-1, \\
& g_{11}=\frac{r^{2}+a^{2} \cos ^{2}(\theta)}{a^{2}+r^{2}-r r_{s}}, \\
& g_{22}=r^{2}+a^{2} \cos ^{2}(\theta), \\
& g_{33}=\sin ^{2}(\theta)\left(\frac{r r_{s} \sin ^{2}(\theta) a^{2}}{r^{2}+a^{2} \cos ^{2}(\theta)}+a^{2}+r^{2}\right), \\
& g_{03}=g_{30}=\frac{a r r_{s} \sin ^{2}(\theta)}{r^{2}+a^{2} \cos ^{2}(\theta)} .
\end{aligned}
$$

Then Eq. (5.1) is

$$
\left.\varepsilon\left\langle T^{\mu \nu}\right\rangle^{\operatorname{div}}\right|_{\text {Kerr }}=\left(\begin{array}{cccc}
\mathcal{A}^{00} & 0 & 0 & \mathcal{A}^{03} \\
0 & \mathcal{A}^{11} & 0 & 0 \\
0 & 0 & \mathcal{A}^{22} & 0 \\
\mathcal{A}^{30} & 0 & 0 & \mathcal{A}^{33}
\end{array}\right)
$$

where

$$
\begin{aligned}
& \mathcal{A}^{00}=-\frac{\mathcal{Y} r_{s}^{2}\left(a^{4}+2 r^{4}+a^{2} r\left(3 r+r_{s}\right)+a^{2}\left(a^{2}+r\left(r-r_{s}\right) \cos (2 \theta)\right)\right.}{\mathcal{X} \mathcal{Z}}, \\
& \mathcal{A}^{11}=\frac{2 \mathcal{X} \mathcal{Y} r_{s}^{2}}{\mathcal{Z}}, \\
& \mathcal{A}^{22}=\frac{2 \mathcal{Y} r_{s}^{2}}{\mathcal{Z}}, \\
& \mathcal{A}^{33}=\frac{\mathcal{Y} r_{s}^{2}\left(a^{2}+2 r\left(r-r_{s}\right)+a^{2} \cos (2 \theta)\right) \csc (\theta)^{2}}{\mathcal{X Z}}, \\
& \mathcal{A}^{03}=\mathcal{A}^{30}=\frac{2 \mathcal{Y} a r r_{s}^{3}}{\mathcal{X} \mathcal{Z}},
\end{aligned}
$$

having defined

$$
\begin{aligned}
\mathcal{X}= & a^{2}+r\left(r-r_{s}\right) \\
\mathcal{Y} \equiv & a^{6} \cos (6 \theta)+10 a^{6}-180 a^{4} r^{2}+240 a^{2} r^{4}+6 a^{4}\left(a^{2}-10 r^{2}\right) \cos (4 \theta) \\
& +15 a^{2}\left(a^{4}-16 a^{2} r^{2}+16 r^{4}\right) \cos (2 \theta)-32 r^{6} \\
\mathcal{Z} \equiv & \left(a^{2}+2 r^{2}+a^{2} \cos (2 \theta)\right)^{7}
\end{aligned}
$$


Of course, if $J=0$, then $a=0$, and the Kerr metric reduces to the Schwarzschild metric; in this case, the same calculations yield

$$
\left.\varepsilon\left\langle T^{\mu \nu}\right\rangle^{\text {div }}\right|_{\text {Schwarzschild }}=\left(\begin{array}{cccc}
\frac{r_{s}^{2}}{2 r^{6}-2 r^{5} r_{s}} & 0 & 0 & 0 \\
0 & \frac{r_{s}^{2}\left(r_{s}-r\right)}{2 r^{7}} & 0 & 0 \\
0 & 0 & -\frac{r_{s}^{2}}{2 r^{8}} & 0 \\
0 & 0 & 0 & -\frac{r_{s}^{2} \csc ^{2}(\theta)}{2 r^{8}}
\end{array}\right) .
$$

\section{Maximally symmetric spaces}

Modern cosmology is based on the hypothesis that on a large enough scale the universe is spatially homogeneous and isotropic. Together, these two assumptions are known as the cosmological principle. As is well known, spaces which are both spatially homogenous and isotropic are maximally symmetric, i.e., they possess the largest possible number of Killing vector fields which in a $n$-dimensional manifold equals $n(n+1) / 2$. It can be shown that the following holds for a maximally symmetric space:

1. The scalar curvature $R$ is a constant;

2. The Ricci tensor is proportional to the metric tensor, i.e.,

$$
R_{\mu \nu}=\frac{1}{n} \operatorname{Rg}_{\mu \nu}
$$

3. The Riemann curvature tensor is given by

$$
R_{\mu \nu \lambda \rho}=\frac{R}{n(n-1)}\left(g_{\mu \lambda} g_{\nu \rho}-g_{\nu \lambda} g_{\mu \rho}\right) .
$$

As a consequence, all terms which involve derivatives in Eq. (4.10) vanish; therefore only nine terms are left in the sum, and Eq. (4.10) takes the simplified form

$$
\begin{aligned}
\varepsilon\left\langle T^{\mu \nu}\right\rangle^{\mathrm{div}}= & g^{\mu \nu}\left(-\frac{23}{180} R_{\alpha \beta} R^{\alpha \beta}+\frac{1}{144} R^{2}-\frac{1}{24} R_{\alpha \beta \gamma \delta} R^{\alpha \gamma \beta \delta}\right. \\
& \left.-\frac{7}{180} R_{\alpha \beta \gamma \delta} R^{\alpha \beta \gamma \delta}\right) \\
& +\frac{17}{180} R_{\alpha \beta} R^{\alpha \mu \nu \beta}+\frac{127}{1080} R^{\mu \alpha \beta \gamma} R_{\alpha \beta \gamma}^{\nu} \\
& -\frac{49}{540} R^{\mu \alpha \beta \gamma} R_{\beta \alpha \gamma}^{\nu}+\frac{19}{180} R_{\alpha}^{\mu} R^{\alpha \nu} \\
& \left.+\frac{1}{18} R R^{\mu \nu}\right] .
\end{aligned}
$$

A crucial example is de Sitter space, which is the maximally symmetric vacuum solution of Einstein's field equations with a positive cosmological constant $\Lambda$ (corresponding to a positive vacuum energy density and negative pressure), actually; the cosmological constant is linked to the Hubble constant by

$$
H=\left(\frac{\Lambda}{3}\right)^{1 / 2} .
$$

It is often useful to coordinatize the space-time in two different ways, depending upon whether one wishes to think of it as an expanding Friedmann-Lemaittre-Robertson-Walker or a static universe with an event horizon. In the former case $(s, \chi, \theta, \phi)$ are used, while $(t, r, \theta, \phi)$ 
coordinates are employed in the latter; the metric components thus become

$$
g_{\mu \nu}=\left(\begin{array}{cccc}
-1 & 0 & 0 & 0 \\
0 & \frac{\cosh ^{2}(H s)}{H^{2}} & 0 & 0 \\
0 & 0 & \frac{\cosh ^{2}(H s) \sin ^{2}(\chi)}{H^{2}} & 0 \\
0 & 0 & 0 & \frac{\cosh ^{2}(H s) \sin ^{2}(\theta) \sin ^{2}(\chi)}{H^{2}}
\end{array}\right),
$$

or

$$
g_{\mu \nu}=\left(\begin{array}{cccc}
H^{2} r^{2}-1 & 0 & 0 & 0 \\
0 & \frac{1}{1-H^{2} r^{2}} & 0 & 0 \\
0 & 0 & r^{2} & 0 \\
0 & 0 & 0 & r^{2} \sin ^{2}(\theta)
\end{array}\right)
$$

Hence, the components of the divergent part of the stress energy-tensor are, in the former case

$$
\left.\varepsilon\left\langle T^{\mu \nu}\right\rangle^{\operatorname{div}}\right|_{\text {de Sitter }(s, \chi, \theta, \phi)}=\left(\begin{array}{cccc}
\mathcal{B}^{00} & 0 & 0 & 0 \\
0 & \mathcal{B}^{11} & 0 & 0 \\
0 & 0 & \mathcal{B}^{22} & 0 \\
0 & 0 & 0 & \mathcal{B}^{33}
\end{array}\right),
$$

where

$$
\begin{aligned}
\mathcal{B}^{00} & =\frac{5}{2} H^{4} \\
\mathcal{B}^{11} & =-\frac{5}{2} H^{6} \operatorname{sech}^{2}(H s), \\
\mathcal{B}^{22} & =-\frac{5}{2} H^{6} \csc ^{2}(\chi) \operatorname{sech}^{2}(H s), \\
\mathcal{B}^{33} & =-\frac{5}{2} H^{6} \csc ^{2}(\theta) \csc ^{2}(\chi) \operatorname{sech}^{2}(H s),
\end{aligned}
$$

while in the latter

$$
\left.\varepsilon\left\langle T^{\mu \nu}\right\rangle^{\operatorname{div}}\right|_{\text {de Sitter }(t, r, \theta, \phi)}=\left(\begin{array}{cccc}
\mathcal{C}^{00} & 0 & 0 & 0 \\
0 & \mathcal{C}^{11} & 0 & 0 \\
0 & 0 & \mathcal{C}^{22} & 0 \\
0 & 0 & 0 & \mathcal{C}^{33}
\end{array}\right),
$$

where

$$
\begin{aligned}
\mathcal{C}^{00} & =\frac{5 H^{4}}{2-2 H^{2} r^{2}}, \\
\mathcal{C}^{11} & =\frac{5}{2} H^{4}\left(-1+H^{2} r^{2}\right), \\
\mathcal{C}^{22} & =-\frac{5 H^{4}}{2 r^{2}} \\
\mathcal{C}^{33} & =-\frac{5 H^{4} \csc ^{2}(\theta)}{2 r^{2}} .
\end{aligned}
$$

\section{Concluding remarks}

In this paper the Feynman propagator for Maxwell's theory in curved space-time has been described by means of the Fock-Schwinger-DeWitt asymptotic expansion; its crucial role has been emphasized, together with the point-splitting method, in the evaluation and regularization of quadratic (in the dynamical field) observables. Among these, there is the stress-energy 
tensor: its matrix element has been derived in terms of second covariant derivatives of the Hadamard Green function of the electromagnetic field. Remarkably, the divergences occurring in $\left\langle T^{\mu \nu}\right\rangle^{\mathrm{vac}}$ and $\left\langle T^{\mu \nu}\right\rangle^{\text {matrix }}$ are identical, therefore regularizing $\left\langle T^{\mu \nu}\right\rangle^{\text {matrix }}$ is equivalent to regularizing $\left\langle T^{\mu v}\right\rangle^{\mathrm{vac}}$. An original computation has been then presented: a concise, explicit formula for the divergent part of the stress-energy tensor. This has been obtained from a careful handling of more than two thousand terms, and in order to perform such a calculation, a program has been written in FORM (see Refs. [16-18]). Such a formula holds for every space-time, and is fully evaluated for some relevant examples: Kerr and Schwarzschild metrics (which are Ricci-flat) and de Sitter metric (which is maximally symmetric); see Refs. $[19,20]$ for an up-todate review. As far as we know, our original results in Eqs. (4.10),(5.1), (5.3), (5.4), (6.4) and (6.6) are not available in the literature (cf. Refs. [25,26]).

In any theory of interacting fields the set of currents that describe the interaction is of fundamental importance; in General Relativity, these currents are the components of the stress-energy tensor, therefore the main problem in developing a quantum field theory in curved space-time is precisely to understand the stress-energy tensor (see Ref. [9]). Compared to the flat space-time case, in a curved background the resulting renormalized stress-energy tensor is covariantly conserved, of course, but it possesses a state-independent anomalous trace (see Refs. [27-30]).

The results obtained are interesting also in the context of effective action theory in curved space-time, whose divergent part is essential to discuss renormalization group equations for the Newton constant and the cosmological constant [24]. Moreover, they can be used to obtain a proper source in the semiclassical gravitational field equations, when doing a back reaction problem, for every background gravitational field.

Acknowledgements R. N. would like to acknowledge the helpful advice that he received from S. M. Christensen. G. E. is grateful to the "Ettore Pancini" Physics Department of Federico II University for hospitality and support.

Funding Open access funding provided by Università degli Studi di Napoli Federico II within the CRUICARE Agreement.

Open Access This article is licensed under a Creative Commons Attribution 4.0 International License, which permits use, sharing, adaptation, distribution and reproduction in any medium or format, as long as you give appropriate credit to the original author(s) and the source, provide a link to the Creative Commons licence, and indicate if changes were made. The images or other third party material in this article are included in the article's Creative Commons licence, unless indicated otherwise in a credit line to the material. If material is not included in the article's Creative Commons licence and your intended use is not permitted by statutory regulation or exceeds the permitted use, you will need to obtain permission directly from the copyright holder. To view a copy of this licence, visit http://creativecommons.org/licenses/by/4.0/.

\section{A $T^{\mu v}$ calculations}

In this appendix the calculations regarding the derivation of (3.3) from (3.7), (3.8), (3.9) are explicitly shown: 
$T_{\text {Maxwell }}^{\mu \nu}$ calculations:

$$
\begin{aligned}
\delta\left(-\frac{1}{4}|\mathrm{~g}|^{1 / 2} F_{\mu \nu} F^{\mu \nu}\right)= & -\frac{1}{4} F_{\mu \nu} F_{\rho \sigma}\left(g^{\mu \rho}\right)\left(g^{\nu \sigma}\right)\left(\delta|\mathrm{g}|^{1 / 2}\right) \\
& -\frac{1}{4} F_{\mu \nu} F_{\rho \sigma}\left(\delta g^{\mu \rho}\right)\left(g^{\nu \sigma}\right)\left(|\mathrm{g}|^{1 / 2}\right) \\
& -\frac{1}{4} F_{\mu \nu} F_{\rho \sigma}\left(g^{\mu \rho}\right)\left(\delta g^{\nu \sigma}\right)\left(|\mathrm{g}|^{1 / 2}\right) \\
= & -\frac{1}{4} F_{\mu \nu} F_{\rho \sigma}\left(g^{\mu \rho}\right)\left(g^{\nu \sigma}\right)\left(\frac{1}{2}|\mathrm{~g}|^{1 / 2} g^{\alpha \beta} \delta g_{\alpha \beta}\right) \\
& -\frac{1}{4} F_{\mu \nu} F_{\rho \sigma}\left(-g^{\mu \alpha} g^{\rho \beta} \delta g_{\alpha \beta}\right)\left(g^{\nu \sigma}\right)\left(|\mathrm{g}|^{1 / 2}\right) \\
& -\frac{1}{4} F_{\mu \nu} F_{\rho \sigma}\left(g^{\mu \rho}\right)\left(-g^{\nu \alpha} g^{\sigma \beta} \delta g_{\alpha \beta}\right)\left(|\mathrm{g}|^{1 / 2}\right) \\
= & -\frac{1}{8} F_{\mu \nu} F^{\mu \nu}|\mathrm{g}|^{1 / 2} g^{\alpha \beta} \delta g_{\alpha \beta} \\
& -\frac{1}{2} F_{\rho}^{\alpha} F^{\rho \beta}|\mathrm{g}|^{1 / 2} \delta g_{\alpha \beta} .
\end{aligned}
$$

Therefore

$$
T_{\text {Maxwell }}^{\mu \nu}=-F_{\rho}^{\mu} F^{\rho v}-\frac{1}{4} F_{\alpha \beta} F^{\alpha \beta} g^{\mu \nu} .
$$

$T_{\text {gauge }}^{\mu \nu}$ calculations:

$$
\begin{aligned}
& \delta\left(-\frac{1}{2}|\mathrm{~g}(x)|^{1 / 2}\left(A_{; \mu}^{\mu}\right)^{2}\right)=-\frac{1}{2}\left(\delta|\operatorname{g}(x)|^{1 / 2}\right)\left(A_{; \mu}^{\mu}\right)^{2} \\
& -\left(|g(x)|^{1 / 2}\right) A_{; \eta}^{\eta}\left[\delta\left(g^{\mu \nu} \nabla_{\nu} A_{\mu}\right)\right] \\
& =-\frac{1}{2}\left(\frac{1}{2}|g(x)|^{1 / 2} g^{\alpha \beta} \delta g_{\alpha \beta}\right)\left(A_{; \mu}^{\mu}\right)^{2} \\
& -\left(|g(x)|^{1 / 2}\right) A_{; \eta}^{\eta}\left[\delta g^{\mu \nu} \nabla_{\nu} A_{\mu}+g^{\mu \nu} \delta\left(\partial_{\nu} A_{\mu}-\Gamma_{\mu \nu}^{\rho} A_{\rho}\right)\right] \\
& =-\frac{1}{4}|g(x)|^{1 / 2}\left(A_{; \mu}^{\mu}\right)^{2} g^{\alpha \beta} \delta g_{\alpha \beta} \\
& +|g(x)|^{1 / 2} A_{; \eta}^{\eta} g^{\mu \alpha} g^{\nu \beta} \delta g_{\alpha \beta} \nabla_{\nu} A_{\mu} \\
& +|g(x)|^{1 / 2} A_{; \eta}^{\eta} g^{\mu v} \delta \Gamma_{\mu \nu}^{\rho} A_{\rho} \\
& =-\frac{1}{4}|g(x)|^{1 / 2}\left(A_{; \mu}^{\mu}\right)^{2} g^{\alpha \beta} \delta g_{\alpha \beta} \\
& +|\mathrm{g}(x)|^{1 / 2} A_{; \eta}^{\eta} \delta g_{\alpha \beta} A^{\alpha ; \beta} \\
& +|\mathrm{g}(x)|^{1 / 2} A_{; \eta}^{\eta} g^{\mu \nu}\left[\frac{1}{2} g^{\rho \alpha}\left(\delta g_{\alpha \nu ; \mu}+\delta g_{\alpha \mu ; \nu}-\delta g_{\mu \nu ; \alpha}\right)\right] A_{\rho} \\
& =-\frac{1}{4}|g(x)|^{1 / 2}\left(A_{; \mu}^{\mu}\right)^{2} g^{\alpha \beta} \delta g_{\alpha \beta} \\
& +|\mathrm{g}(x)|^{1 / 2} A_{; \eta}^{\eta} \delta g_{\alpha \beta} A^{\alpha ; \beta} \\
& +|g(x)|^{1 / 2} A_{; \eta}^{\eta} g^{\mu \nu} A^{\alpha} \delta g_{\alpha \nu ; \mu}-\frac{1}{2}|g(x)|^{1 / 2} A_{; \eta}^{\eta} A^{\alpha} g^{\mu \nu} \delta g_{\mu \nu ; \alpha} .
\end{aligned}
$$

Integrating by parts on the last line, one obtains 


$$
\begin{aligned}
\delta\left(-\frac{1}{2}|\mathrm{~g}(x)|^{1 / 2}\left(A_{; \mu}^{\mu}\right)^{2}\right)= & -\frac{1}{4}|\mathrm{~g}(x)|^{1 / 2}\left(A_{; \mu}^{\mu}\right)^{2} g^{\alpha \beta} \delta g_{\alpha \beta} \\
& +|\mathrm{g}(x)|^{1 / 2} A_{; \eta}^{\eta} A^{\alpha ; \beta} \delta g_{\alpha \beta} \\
& -|\mathrm{g}(x)|^{1 / 2} A_{; \eta \mu}^{\eta} g^{\mu \nu} A^{\alpha} \delta g_{\alpha \nu}-|\mathrm{g}(x)|^{1 / 2} A_{; \eta}^{\eta} g^{\mu \nu} A_{; \mu}^{\alpha} \delta g_{\alpha \nu} \\
& +\frac{1}{2}|\mathrm{~g}(x)|^{1 / 2} A_{; \eta \alpha}^{\eta} A^{\alpha} g^{\mu v} \delta g_{\mu \nu}+\frac{1}{2}|\mathrm{~g}(x)|^{1 / 2} A_{; \eta}^{\eta} A_{; \alpha}^{\alpha} g^{\mu \nu} \delta g_{\mu \nu} \\
= & \left(\frac{1}{4}\left(A_{; \mu}^{\mu}\right)^{2}+\frac{1}{2} A_{; \nu \rho}^{\mu} A^{\rho}\right)|\mathrm{g}(x)|^{1 / 2} g^{\alpha \beta} \delta g_{\alpha \beta} \\
& -\frac{1}{2}\left(A_{\eta}^{; \eta \alpha} A^{\beta}+A_{\eta} ; \eta \beta A^{\alpha}\right)|\mathrm{g}(x)|^{1 / 2} \delta g_{\alpha \beta} .
\end{aligned}
$$

Therefore

$$
T_{\text {gauge }}^{\mu \nu}=\left(\frac{1}{2}\left(A_{; \alpha}^{\alpha}\right)^{2}+A_{; \alpha \beta}^{\alpha} A^{\beta}\right) g^{\mu \nu}-\left(A_{\alpha}^{; \alpha \mu} A^{\nu}+A_{\alpha}^{; \alpha \nu} A^{\mu}\right) .
$$

$T_{\text {ghost }}^{\mu \nu}$ calculations:

$$
\begin{aligned}
\delta\left(-|\mathrm{g}(x)|^{1 / 2} \chi \square \psi\right)= & -\delta\left(|\mathrm{g}(x)|^{1 / 2}\right) \chi \square \psi-|\mathrm{g}(x)|^{1 / 2} \chi \delta(\square \psi) \\
= & \left.-\frac{1}{2}|\mathrm{~g}(x)|^{1 / 2}\right) \chi \square \psi g^{\mu \nu} \delta g_{\mu \nu} \\
& -|\mathrm{g}(x)|^{1 / 2} \chi \delta\left(g^{\nu \mu} \partial_{\mu} \partial_{\nu} \psi-g^{\nu \mu} \Gamma_{\mu \nu}^{\rho} \partial_{\rho} \psi\right) \\
= & -\frac{1}{2}|\mathrm{~g}(x)|^{1 / 2} \chi \square \psi g^{\mu \nu} \delta g_{\mu \nu} \\
& +|\mathrm{g}(x)|^{1 / 2} \chi g^{\nu \alpha} g^{\mu \beta} \delta g_{\alpha \beta} \partial_{\mu} \partial_{\nu} \psi \\
& -|\mathrm{g}(x)|^{1 / 2} \chi g^{\nu \alpha} g^{\mu \beta} \delta g_{\alpha \beta} \Gamma_{\mu \nu}^{\rho} \partial_{\rho} \psi \\
& +|\mathrm{g}(x)|^{1 / 2} \chi g^{\nu \mu} \delta \Gamma_{\mu \nu}^{\rho} \partial_{\rho} \psi \\
= & -\frac{1}{2}|\mathrm{~g}(x)|^{1 / 2} \chi \square \psi g^{\mu \nu} \delta g_{\mu \nu} \\
& +|\mathrm{g}(x)|^{1 / 2} \chi g^{\nu \alpha} g^{\mu \beta} \delta g_{\alpha \beta} \psi_{; \nu \mu} \\
& +|\mathrm{g}(x)|^{1 / 2} \chi g^{\nu \mu}\left(\frac{1}{2} g^{\rho \eta}\left(\nabla_{\mu} \delta g_{\nu \eta}+\nabla_{\nu} \delta g_{\mu \eta}-\nabla_{\eta} \delta g_{\mu \nu}\right)\right) \partial_{\rho} \psi \\
= & -\frac{1}{2}|g(x)|^{1 / 2} \chi \square \psi g^{\mu \nu} \delta g_{\mu \nu} \\
& +|\mathrm{g}(x)|^{1 / 2} \chi g^{\nu \alpha} g^{\mu \beta} \delta g_{\alpha \beta} \psi_{; \nu \mu} \\
& +|\mathrm{g}(x)|^{1 / 2} \chi g^{\nu \mu} g^{\rho \eta} \delta g_{\nu \eta ; \mu} \partial_{\rho} \psi-\frac{1}{2}|\mathrm{~g}(x)|^{1 / 2} \chi g^{\nu \mu} g^{\rho \eta} \delta g_{\mu \nu ; \eta} \partial_{\rho} \psi .
\end{aligned}
$$

Upon integrating by parts on the last line, one obtains

$$
\begin{aligned}
& =-\frac{1}{2}|g(x)|^{1 / 2} \chi \square \psi g^{\mu v} \delta g_{\mu \nu} \\
& +|g(x)|^{1 / 2} \chi g^{\nu \alpha} g^{\mu \beta} \delta g_{\alpha \beta} \psi_{; v \mu} \\
& -|g(x)|^{1 / 2} \chi g^{\nu \mu} g^{\rho \eta} \delta g_{\nu \eta} \psi_{; \rho \mu}-|g(x)|^{1 / 2} \chi_{; \mu} g^{\nu \mu} g^{\rho \eta} \delta g_{\nu \eta} \psi_{; \rho} \\
& +\frac{1}{2}|g(x)|^{1 / 2} \chi_{; \eta} g^{\nu \mu} g^{\rho \eta} \delta g_{\mu \nu} \psi_{; \rho}+\frac{1}{2}|g(x)|^{1 / 2} \chi g^{\nu \mu} g^{\rho \eta} \delta g_{\mu \nu} \psi_{; \rho} \eta \\
& =-\frac{1}{2}|g(x)|^{1 / 2} \chi \psi_{; \alpha}{ }^{\alpha} g^{\mu v} \delta g_{\mu \nu} \\
& +|\mathrm{g}(x)|^{1 / 2} \chi \psi^{; \alpha \beta} \delta g_{\alpha \beta} \\
& -|\mathrm{g}(x)|^{1 / 2} \chi \psi^{; \eta \nu} \delta g_{\nu \eta}-|\mathrm{g}(x)|^{1 / 2} \chi^{; \mu} \psi^{; \eta} \delta g_{\mu \eta} \\
& +\frac{1}{2}|g(x)|^{1 / 2} \chi_{; \eta} \psi^{; \eta} g^{\nu \mu} \delta g_{\mu \nu}+\frac{1}{2}|g(x)|^{1 / 2} \chi g^{\nu \mu} \psi_{; \eta}{ }^{\eta} \delta g_{\mu \nu} \\
& =\frac{1}{2}|g(x)|^{1 / 2} \chi_{; \alpha} \psi^{; \alpha} g^{\mu v} \delta g_{\mu \nu}-\frac{1}{2}|g(x)|^{1 / 2}\left(\chi^{; \mu} \psi^{; v}+\chi^{; v} \psi^{; \mu}\right) \delta g_{\mu \nu} \text {. }
\end{aligned}
$$


Therefore

$$
T_{\text {ghost }}^{\mu \nu}=\chi_{; \alpha} \psi^{; \alpha} g^{\mu \nu}-\left(\chi^{; \mu} \psi^{; v}+\chi^{; v} \psi^{; \mu}\right) .
$$

\section{B DeWitt's beautiful result}

\section{B.1 "In" and "Out" regions, Bogoliubov coefficients}

In this appendix the proof of the following fundamental result will be outlined:

$$
\left\langle T^{\mu \nu}\right\rangle^{\text {vac }}=\left\langle T^{\mu \nu}\right\rangle^{\text {matrix }}+\left\langle T^{\mu v}\right\rangle^{\text {finite }},
$$

where $\left\langle T^{\mu \nu}\right\rangle^{\text {finite }}$ vanishes when there is no particle annihilation, is always finite, and satisfies the conservation equation $\left\langle T^{\mu \nu}\right\rangle_{; \nu}^{\text {finite }}=0$.

Let us assume that space-time has two causally connected stationary regions, an "in" region and an "out" region, each possessing complete Cauchy hypersurfaces and a timelike Killing vector, which makes it possible to define positive and negative frequencies. Let us consider a free theory, i.e., a theory whose action functional $S$ is quadratic in the (real) dynamical field; then the equation of motion is given in terms of a linear, self-adjoint, differential operator $F$, to which a conserved inner product on the space of solutions is associated. Hence, given a complete set $\left\{u_{i}\right\}$ of normalised basis solutions which contain only positive frequencies, the field solution can be expanded in the form

$$
\phi(x)=\sum_{i}\left(a_{i} u_{i}(x)+a_{i}^{\dagger} u_{i}^{*}(x)\right) .
$$

By using the canonical (anti)commutation relations, or, in a more elegant and manifestly covariant way, by using the Peierls [31] definition of (anti-)commutator, it is easy to see that the operator coefficients in the expansion satisfy the (anti-)commutation relations

$$
\begin{aligned}
& {\left[a_{i}, a_{j}^{\dagger}\right]_{ \pm}=\underline{\delta}_{i j},} \\
& {\left[a_{i}, a_{j}\right]_{ \pm}=0 .}
\end{aligned}
$$

This operator algebra serves in the traditional fashion to define a Fock space and a "vacuum" state:

$$
a_{i}|\mathrm{vac}\rangle=0 .
$$

In our case, there are two sets $\left\{u_{i}\right.$ in $\},\left\{u_{i}\right.$ out $\}$ of normalised basis solutions which contain only positive frequencies, associated with the "in" and "out" regions, respectively; they will be connected by a Bogoliubov transformation

$$
u_{i \text { out }}=\sum_{j}\left(\alpha_{i j} u_{j \text { in }}+\beta_{i j} u_{j \text { in }}^{*}\right)
$$

then we have

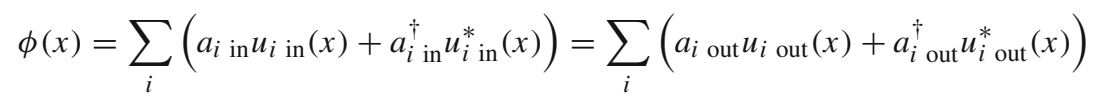

and the "vacuum" state vectors are defined by

$$
\begin{aligned}
& \left.a_{i \text { in }} \mid \text { in, vac }\right\rangle=0, \\
& \left.a_{i \text { out }} \mid \text { out, vac }\right\rangle=0 .
\end{aligned}
$$


The annihilation operators in the "in" and "out" regions are related by

$$
\begin{aligned}
& a_{i \text { out }}=\sum_{j}\left(\alpha_{i j}^{*} a_{j \text { in }}-\beta_{i j}^{*} a_{j \text { in }}^{\dagger}\right), \\
& a_{i \text { in }}=\sum_{j}\left(\alpha_{j i} a_{j \text { out }}-\beta_{j i}^{*} a_{j \text { out }}^{\dagger}\right) .
\end{aligned}
$$

B.2 Creation and annihilation amplitudes, vacuum-to-vacuum amplitude, relations of its divergencies with those of $T_{\mu \nu}$

Of particular importance in quantum cosmology are the many-particle production and annihilation amplitudes:

$$
\begin{aligned}
i^{n / 2} V_{i_{1} \ldots i_{n}} & \left.\equiv e^{-i W}\left\langle\text { out }, i_{1} \ldots i_{n}\right| \text { in, vac }\right\rangle, \\
i^{n / 2} \Lambda_{i_{1} \ldots i_{n}} & \left.\equiv e^{-i W}\langle\text { out, vac }| \text { in }, i_{1} \ldots i_{n}\right\rangle,
\end{aligned}
$$

where

$$
\begin{aligned}
e^{i W} & \equiv\langle\text { out, vac }| \text { in, vac }\rangle \\
\left.\mid \text { in }, i_{1} \ldots i_{n}\right\rangle & \left.\equiv a_{i_{1} \text { in }}^{\dagger} \ldots a_{i_{n} \text { in }}^{\dagger} \mid \text { in, vac }\right\rangle \\
\left.\mid \text { out }, i_{1} \ldots i_{n}\right\rangle & \left.\equiv a_{i_{1} \text { out }}^{\dagger} \ldots a_{i_{n} \text { out }}^{\dagger} \mid \text { out, vac }\right\rangle
\end{aligned}
$$

Assuming unit normalization for the "in" and "out" vacuum state vectors, one may write

$$
\begin{aligned}
\mid \text { in }, \text { vac }\rangle & \left.=e^{i W} \sum_{n=0}^{+\infty} \frac{i^{n / 2}}{n !} \sum_{j_{1} \ldots j_{n}} V_{j_{1} \ldots j_{n}} \mid \text { out }, j_{1} \ldots j_{n}\right\rangle \\
\mid \text { out }, \text { vac }\rangle & \left.=e^{-i W^{*}} \sum_{n=0}^{+\infty} \frac{(-i)^{n / 2}}{n !} \sum_{j_{1} \ldots j_{n}} \Lambda_{j_{1} \ldots j_{n}}{ }^{*} \mid \text { in }, j_{1} \ldots j_{n}\right\rangle .
\end{aligned}
$$

It can be easily proven that the production amplitudes $V_{i_{1} \ldots i_{n}}$ and the annihilation amplitudes $\Lambda_{i_{1} \ldots i_{n}}$ obey

$$
\begin{aligned}
& V_{i_{1} \ldots i_{n}}= \begin{cases}0 & n \text { odd } \\
\sum_{p} V_{i_{1} i_{2}} \ldots V_{i_{n-1} i_{n}} & n \text { even }\end{cases} \\
& \Lambda_{i_{1} \ldots i_{n}}= \begin{cases}0 & n \text { odd, } \\
\sum_{p} \Lambda_{i_{1} i_{2}} \ldots \Lambda_{i_{n-1} i_{n}} & n \text { even },\end{cases}
\end{aligned}
$$

where $\sum_{p}$ denotes a summation over the $\frac{n !}{2^{n / 2}(n / 2) !}$ distinct pairings of the labels $i_{1}, \ldots, i_{n}$. The previous equations reveal that particle production and annihilation processes are composed of individual pair creation and annihilation events.

Consider now an infinitesimal change $\delta g_{\mu \nu}$ in the metric tensor; it yields a change $\delta S$ in the action functional for the field $\phi$; if the support of $\delta g_{\mu \nu}$ is confined to the space-time region between the "in" and "out" regions then, by Schwinger variational principle, it follows that:

$$
\begin{aligned}
\delta W & \left.=-i e^{-i W} \delta e^{i W}=-i e^{-i W} \delta\langle\text { out }, \text { vac }| \text { in }, \text { vac }\right\rangle \\
& =e^{-i W}\langle\text { out, } \operatorname{vac}|\delta S| \text { in, vac }\rangle,
\end{aligned}
$$


which implies

$$
\frac{2}{\sqrt{\mid \mathrm{g}} \mid} \frac{\delta W}{\delta g_{\mu \nu}}=e^{-i W}\left\langle\text { out, vac }\left|T^{\mu \nu}\right| \text { in, vac }\right\rangle \equiv\left\langle T^{\mu \nu}\right\rangle^{\text {matrix }} .
$$

Introduce now the following notation, in which integration over space-time coordinates is suppressed:

$$
\begin{aligned}
S[\phi] & =\frac{1}{2} \phi F \phi, \\
\frac{\delta S}{\delta \phi}[\phi] & =F \phi .
\end{aligned}
$$

Then a more precise notation for the stress energy tensor can be introduced:

$$
\begin{aligned}
T^{\mu \nu}(\chi, \psi) & \equiv \chi \frac{\delta F}{\delta g_{\mu \nu}} \psi, \\
\frac{2}{\sqrt{|\mathrm{g}|}} \frac{\delta S}{\delta g_{\mu \nu}}[\phi] & =\phi \frac{\delta F}{\delta g_{\mu \nu}} \phi \equiv T^{\mu \nu}(\phi, \phi) ;
\end{aligned}
$$

therefore Eq. (B.22) is

$$
\left\langle T^{\mu \nu}\right\rangle^{\text {matrix }}=e^{-i W}\left\langle\text { out, } \operatorname{vac}\left|T^{\mu \nu}(\phi, \phi)\right| \text { in, vac }\right\rangle .
$$

Using the field expansion and the creation/annihilation amplitudes, one obtains

$$
\begin{aligned}
& \left\langle T^{\mu \nu}\right\rangle^{\text {matrix }} \\
& =\sum_{i} T^{\mu \nu}\left(u_{i \text { in }}, u_{i \text { in }}{ }^{*}\right)+i \sum_{i, j} \Lambda_{i j} T^{\mu \nu}\left(u_{i \text { in }}{ }^{*}, u_{j \text { in }}{ }^{*}\right) ;
\end{aligned}
$$

The first term is

$$
\sum_{i} T^{\mu \nu}\left(u_{i \text { in }}, u_{i \text { in }}{ }^{*}\right)=\left\langle\text { in, } \operatorname{vac}\left|T^{\mu \nu}(\phi, \phi)\right| \text { in, vac }\right\rangle=\left\langle T^{\mu \nu}(\phi, \phi)\right\rangle^{\mathrm{vac}} \equiv\left\langle T^{\mu \nu}\right\rangle^{\mathrm{vac}},
$$

while the second term is zero when there is no particle annihilation (because of the presence of $\Lambda_{i j}$ ) and is always finite; let us show that it satisfies the conservation equation; it suffices to note that

$$
T_{; v}^{\mu v}(\phi, \phi)=0 \text { for every solution } \phi
$$

and that

$$
\begin{aligned}
& T^{\mu v}\left(u_{i \text { in }}{ }^{*}+u_{j \text { in }}{ }^{*}, u_{i \text { in }}{ }^{*}+u_{j \text { in }}{ }^{*}\right) \\
& =T^{\mu v}\left(u_{i \text { in }}{ }^{*}, u_{i \text { in }}{ }^{*}\right)+T^{\mu v}\left(u_{j \text { in }}{ }^{*}, u_{j \text { in }}{ }^{*}\right)+2 T^{\mu v}\left(u_{i \text { in }}{ }^{*}, u_{j \text { in }}{ }^{*}\right) ;
\end{aligned}
$$

therefore (B.30) implies

$$
T_{; v}^{\mu v}\left(u_{i \text { in }}{ }^{*}, u_{j \text { in }}{ }^{*}\right)=0,
$$

i.e., the conservation equation. Hence it is proven that

$$
\left\langle T^{\mu \nu}\right\rangle^{\text {vac }}=\left\langle T^{\mu \nu}\right\rangle^{\text {matrix }}+\left\langle T^{\mu \nu}\right\rangle^{\text {finite }},
$$

where $\left\langle T^{\mu \nu}\right\rangle^{\text {finite }}$ is zero when there is no particle annihilation, is always finite, and satisfies the conservation equation $\left\langle T^{\mu \nu}\right\rangle_{; \nu}^{\text {finite }}=0$; it can be expressed as

$$
\left\langle T^{\mu v}\right\rangle^{\text {finite }}=-i \sum_{i, j} \Lambda_{i j} T^{\mu v}\left(u_{i \text { in }}{ }^{*}, u_{j \text { in }}{ }^{*}\right) .
$$




\section{World function: coincidence limits}

C.1 General equations satisfied by the covariant derivatives of the world function

Following Synge in Ref. [32], we will show a method to calculate the coincidence limits of the covariant derivatives of the world function, up to order six; these quantities are crucial in the evaluation of the divergent part of the stress-energy tensor. First, one needs to recall the fundamental equations satisfied by the world function:

$$
\begin{aligned}
\sigma_{; \mu} \sigma^{; \mu} & =2 \sigma, \\
\sigma_{; \mu \nu \rho}-\sigma_{; \mu \rho v} & =-R_{\mu \lambda \nu \rho} \sigma^{; \lambda} .
\end{aligned}
$$

Differentiating the first equation, and indicating with a subscript that a certain index is free, one obtains

$$
\begin{aligned}
& \sigma_{; \alpha_{1} \mu} \sigma^{; \mu}=\sigma_{; \alpha_{1}}, \\
& \sigma_{; \alpha_{1} \mu \alpha_{2}} \sigma^{; \mu}+\sigma_{; \alpha_{1} \mu} \sigma_{\alpha_{2}}^{; \mu}=\sigma_{; \alpha_{1} \alpha_{2}}, \\
& \sigma_{; \alpha_{1} \mu \alpha_{2} \alpha_{3}} \sigma^{; \mu}+\sigma_{; \alpha_{1} \mu \alpha_{2}} \sigma_{\alpha_{3}}^{; \mu}+\sigma_{; \alpha_{1} \mu \alpha_{3}} \sigma_{\alpha_{2}}^{; \mu}+\sigma_{; \alpha_{1} \mu} \sigma_{\alpha_{2} \alpha_{3}}^{; \mu} \\
& \quad=\sigma_{; \alpha_{1} \alpha_{2} \alpha_{3}},
\end{aligned}
$$

while differentiating the second one, one obtains

$$
\begin{aligned}
\sigma_{; \alpha_{1} \alpha_{2} \alpha_{3} \alpha_{4}}-\sigma_{; \alpha_{1} \alpha_{3} \alpha_{2} \alpha_{4}}= & -R_{\alpha_{1} \lambda \alpha_{2} \alpha_{3} ; \alpha_{4}} \sigma^{; \lambda}-R_{\alpha_{1} \lambda \alpha_{2} \alpha_{3}} \sigma_{\alpha_{4}}^{; \lambda}, \\
\sigma_{; \alpha_{1} \alpha_{2} \alpha_{3} \alpha_{4} \alpha_{5}}-\sigma_{; \alpha_{1} \alpha_{3} \alpha_{2} \alpha_{4} \alpha_{5}}= & -R_{\alpha_{1} \lambda \alpha_{2} \alpha_{3} ; \alpha_{4} \alpha_{5}} \sigma^{; \lambda}-R_{\alpha_{1} \lambda \alpha_{2} \alpha_{3} ; \alpha_{4}} \sigma_{\alpha_{5}}^{; \lambda} \\
& -R_{\alpha_{1} \lambda \alpha_{2} \alpha_{3} ; \alpha_{5}} \sigma_{\alpha_{4}}-R_{\alpha_{1} \lambda \alpha_{2} \alpha_{3}} \sigma_{\alpha_{4} \alpha_{5}}^{; \lambda} \\
\sigma_{; \alpha_{1} \alpha_{2} \alpha_{3} \alpha_{4} \alpha_{5} \alpha_{6}}-\sigma_{; \alpha_{1} \alpha_{3} \alpha_{2} \alpha_{4} \alpha_{5} \alpha_{6}}= & -R_{\alpha_{1} \lambda \alpha_{2} \alpha_{3} ; \alpha_{4} \alpha_{5} \alpha_{6}} \sigma^{; \lambda}-R_{\alpha_{1} \lambda \alpha_{3} \alpha_{2} ; \alpha_{4} \alpha_{5}} \sigma_{\alpha_{6}}^{; \lambda} \\
& -R_{\alpha_{1} \lambda \alpha_{2} \alpha_{3} ; \alpha_{4} \alpha_{6}} \sigma^{; \lambda}{ }_{\alpha_{5}}-R_{\alpha_{1} \lambda \alpha_{3} \alpha_{2} ; \alpha_{4}} \sigma_{\alpha_{5} \alpha_{6}}^{; \lambda} \\
& -R_{\alpha_{1} \lambda \alpha_{2} \alpha_{3} ; \alpha_{5} \alpha_{6}} \sigma_{\alpha_{4}}^{; \lambda}-R_{\alpha_{1} \lambda \alpha_{2} \alpha_{3} ; \alpha_{5}} \sigma_{\alpha_{4} \alpha_{6}}^{; \lambda} \\
& -R_{\alpha_{1} \lambda \alpha_{2} \alpha_{3} ; \alpha_{6}} \sigma_{\alpha_{4} \alpha_{5}}-R_{\alpha_{1} \lambda \alpha_{2} \alpha_{3}} \sigma_{\alpha_{4} \alpha_{5} \alpha_{6}} .
\end{aligned}
$$

For the sake of readability, the following notation will be introduced: when an index is not a summation index, it will be indicated with a number, e.g.

$$
\sigma_{; \alpha_{1} \alpha_{2} \lambda \alpha_{3} \alpha_{4} \alpha_{5}} \equiv \sigma_{; 12 \lambda 345},
$$

and the Riemann tensor, with its derivatives, will be indicated through the sequence of its indices between parentheses, e.g.

$$
R_{\alpha_{1} \lambda \alpha_{2} \alpha_{3} ; \alpha_{6}} \equiv(1, \lambda, 2,3 ; 6) .
$$

Then the equations which result from differentiations of (C.1) can be put in the form

$$
\sigma_{; 123 \ldots l}=\sum \sigma_{; 1 \lambda a_{2} a_{3} \ldots a_{k}} \sigma^{; \lambda} a_{k+1} \ldots a_{l}
$$

where the summation extends over all expressions for which $a_{2}, a_{3}, \ldots, a_{k}$ is a set selected in order from $2, \ldots, l$ and $a_{k+1}, \ldots, a_{l}$ is the remainder of the set $2, \ldots, l$ in order, and $k$ takes all values from 2 to $l$. The equations which follow from differentiations on (C.2) take the form

$$
\sigma_{; 123 \ldots l}-\sigma_{; 132 \ldots l}=-\sum\left(1, \lambda, 2,3 ; b_{4}, \ldots, b_{k}\right) \sigma_{b_{k+1} \ldots b_{l}}^{; \lambda},
$$


where the summation extends over all expressions for which $b_{4}, \ldots, b_{k}$ is a set selected in order from $4, \ldots, l$ and $b_{k+1}, \ldots, b_{l}$ is the remainder of the set $4, \ldots, l$ in order, and $k$ takes all values from 4 to $l$.

C.2 Coincidence limits of covariant derivatives

Dividing (C.3) by $\sigma$, one obtains

$$
\sigma_{; 1 \mu} t^{\mu}=t_{1},
$$

where $t$ is the unit tangent vector along the geodesic; then the coincidence limit reads

$$
\left[\sigma_{; 12}\right]=g_{12} .
$$

From (C.4) and (C.6) one has

$$
\left[\sigma_{; 123}\right]+\left[\sigma_{; 132}\right]=0=\left[\sigma_{; 123}\right]-\left[\sigma_{; 132}\right]
$$

which implies

$$
\left[\sigma_{; 123}\right]=0 .
$$

For the next order, by (C.5) and (C.7) one has

$$
\begin{aligned}
& {\left[\sigma_{; 1234}\right]+\left[\sigma_{; 1423}\right]+\left[\sigma_{; 1324}\right]=0} \\
& {\left[\sigma_{; 1234}\right]-\left[\sigma_{; 1323}\right]=-(1,4,2,3) .}
\end{aligned}
$$

By (C.16) we convert (C.15) into

$$
\left[\sigma_{; 1243}\right]+2\left[\sigma_{; 1234}\right]+(1,3,2,4)+(1,4,2,3)=0 ;
$$

Interchanging 3 and 4 and subtracting, we get

$$
\left[\sigma_{; 1234}\right]=\left[\sigma_{; 1243}\right],
$$

and thus (C.17) gives

$$
\left[\sigma_{; 1234}\right]=-\frac{1}{3}((1,3,2,4)+(1,4,2,3)),
$$

or

$$
\left[\sigma_{; 1234}\right]=-\frac{1}{3} \mathcal{P}_{3,4}(1,3,2,4),
$$

where $\mathcal{P}_{3,4}$ denotes the sum of expressions obtained by permutation of 3,4 .

For the fifth order, by (C.9) and (C.10),

$$
\begin{aligned}
& {\left[\sigma_{; 15234}\right]+\left[\sigma_{; 14235}\right]+\left[\sigma_{; 13245}\right]+\left[\sigma_{; 12345}\right]=0,} \\
& {\left[\sigma_{; 12345}\right]-\left[\sigma_{; 13245}\right]=-(1,4,2,3 ; 5)-(1,5,2,3 ; 4) .}
\end{aligned}
$$

By (C.22) we convert (C.21) into

$$
\begin{aligned}
& {\left[\sigma_{; 12534}\right]+\left[\sigma_{; 12435}\right]+2\left[\sigma_{; 12345}\right]} \\
& \quad+(1,4,2,3 ; 5)+(1,5,2,3 ; 4)+(1,3,2,4 ; 5) \\
& \quad+(1,5,2,4 ; 3)+(1,3,2,5 ; 4)+(1,4,2,5 ; 3)=0 .
\end{aligned}
$$

Interchanging 4 and 5, and subtracting, we get

$$
\left[\sigma_{; 12345}\right]=\left[\sigma_{; 12354}\right]
$$


interchanging 3 and 4 in (C.23), subtracting, and using (C.24), we get

$$
\left[\sigma_{; 12345}\right]=\left[\sigma_{; 12435}\right]
$$

thus (C.23) becomes

$$
\begin{aligned}
{\left[\sigma_{; 12345}\right]=} & -\frac{1}{4}((1,3,2,4 ; 5)+(1,3,2,5 ; 4)+(1,4,2,3 ; 5) \\
& +(1,4,2,5 ; 3)+(1,5,2,3 ; 4)+(1,5,2,4 ; 3)) .
\end{aligned}
$$

or

$$
\left[\sigma_{; 12345}\right]=-\frac{1}{4} \mathcal{P}_{3,4,5}(1,3,2,4 ; 5) .
$$

For the sixth order we have, by (C.9),

$$
\begin{aligned}
& {\left[\sigma_{; 162345}\right]+\left[\sigma_{; 152346}\right]+\left[\sigma_{; 142356}\right]} \\
& \quad+\left[\sigma_{; 132456}\right]+\left[\sigma_{; 123456}\right]+\left[H_{123456}\right]=0,
\end{aligned}
$$

where

$$
\begin{aligned}
& {\left[H_{123456}\right]} \\
& =\left[\sigma_{; 1 \lambda 23}\right]\left[\sigma_{456}^{; \lambda}\right]+\left[\sigma_{; 1 \lambda 24}\right]\left[\sigma_{356}^{; \lambda}\right]+\left[\sigma_{; 1 \lambda 25}\right]\left[\sigma_{346}^{; \lambda}\right]+\left[\sigma_{; 1 \lambda 26}\right]\left[\sigma_{345}^{; \lambda}\right] \\
& \quad+\left[\sigma_{; 1 \lambda 34}\right]\left[\sigma_{256}^{; \lambda}\right]+\left[\sigma_{; 1 \lambda 35}\right]\left[\sigma_{246}^{; \lambda}\right]+\left[\sigma_{; 1 \lambda 36}\right]\left[\sigma_{245}^{; \lambda}\right] \\
& \quad+\left[\sigma_{; 1 \lambda 45}\right]\left[\sigma_{236}^{; \lambda}\right]+\left[\sigma_{; 1 \lambda 46}\right]\left[\sigma_{235}^{; \lambda}\right] \\
& \quad+\left[\sigma_{; 1 \lambda 56}\right]\left[\sigma_{234}^{; \lambda}\right]
\end{aligned}
$$

is a tensor whose value is known by (C.20); from (C.10) we have

$$
\begin{aligned}
& {\left[\sigma_{; 123456}\right]-\left[\sigma_{; 132456}\right]} \\
& \quad=-(1,4,2,3 ; 5,6)-(1,5,2,3 ; 4,6) \\
& \quad-(1,6,2,3 ; 4,5)-(1, \lambda, 2,3)\left[\sigma_{4,5,6}^{; \lambda}\right]
\end{aligned}
$$

By means of this equation we convert (C.28) into

$$
\begin{aligned}
& {\left[\sigma_{; 126345}\right]+\left[\sigma_{; 125346}\right]+\left[\sigma_{; 124356}\right]+2\left[\sigma_{; 123456}\right]+\left[H_{123456}\right]} \\
& \quad+(1,4,2,3 ; 5,6)+(1,5,2,3 ; 4,6)+(1,6,2,3 ; 4,5)+(1, \lambda, 2,3) \sigma_{456}^{; \lambda} \\
& \quad+(1,3,2,4 ; 5,6)+(1,5,2,4 ; 3,6)+(1,6,2,4 ; 3,5)+(1, \lambda, 2,4) \sigma_{356}^{; \lambda} \\
& \quad+(1,3,2,5 ; 4,6)+(1,4,2,5 ; 3,6)+(1,6,2,5 ; 3,4)+(1, \lambda, 2,5) \sigma_{346}^{; \lambda} \\
& \quad+(1,3,2,6 ; 4,5)+(1,4,2,6: 3,5)+(1,5,2,3 ; 6,4)+(1, \lambda, 2,6) \sigma_{345}^{; \lambda} \\
& =0 .
\end{aligned}
$$

If we employ the symbol $I$ to denote the operation of interchanging numerals, so that $I(3,4)$, for example, denotes an interchange of 3 and 4 , the previous equation may be written

$$
\begin{aligned}
& \{2+I(3,4)+I(4,5) I(3,4)+I(5,6) I(4,5) I(3,4)\}\left[\sigma_{; 123456}\right] \\
& \quad+\{1+I(3,4)+I(4,5) I(3,4)+I(5,6) I(4,5) I(3,4)\}\left[L_{123456}\right] \\
& \quad+\left[H_{123456}\right]=0
\end{aligned}
$$

where $\left[L_{123456}\right]$ denotes the second line of (C.31). 
Operating with $\{1-I(5,6)\}$ on (C.31), and using (C.18), we get

$$
\begin{aligned}
& \{2+I(3,4)\}\{1-I(5,6)\}\left[\sigma_{; 123456}\right] \\
& \quad+\{1+I(3,4)\}\{1-I(5,6)\}(1,3,2,4 ; 5,6)=0 .
\end{aligned}
$$

Since

$$
I(3,4) I(3,4)=1,
$$

we have

$$
\begin{aligned}
& \{2-I(3,4)\}\{2+I(3,4)\}=3, \\
& \{2-I(3,4)\}\{1+I(3,4)\}=1+I(3,4),
\end{aligned}
$$

and therefore operation on (C.33) with $\{2-I(3,4)\}$ gives

$$
\{1-I(5,6)\}\left[\sigma_{; 123456}\right]=-\frac{1}{3}\{1+I(3,4)\}\{1-I(5,6)\}(1,3,2,4 ; 5,6) .
$$

Operation on (C.31) with $\{1-I(4,5)\}$ gives

$$
\begin{aligned}
& \{1-I(4,5)\}\left[\sigma_{; 126345}\right]+2\{1-I(4,5)\}\left[\sigma_{; 123456}\right]+\{1-I(4,5)\}\left[H_{123456}\right] \\
& \quad+\{1+I(3,6)\}\{1-I(4,5)\}(1,3,2,6 ; 4,5) \\
& \quad+\{1-I(4,5)\}(1, \lambda, 2,3)\left[\sigma_{456}^{; \lambda}\right]=0
\end{aligned}
$$

in which the first term may be evaluated by applying to (C.35) the substitution

$$
\begin{array}{llllll}
1 & 2 & 3 & 4 & 5 & 6 \\
1 & 2 & 6 & 3 & 4 & 5
\end{array}
$$

while the term in $[H]$ is, by (C.29)

$$
\{1-I(4,5)\}\left[\sigma_{; 1 \lambda 23}\right]\left[\sigma_{456}^{; \lambda}\right] .
$$

Thus (C.36) may be written

$$
\begin{aligned}
& \{1-I(4,5)\}\left[\sigma_{; 123456}\right]=-\frac{1}{3}\{1+I(3,6)\}\{1-I(4,5)\}(1,3,2,6 ; 4,5) \\
& -\frac{1}{2}\{1-I(4,5)\}\left((1, \lambda, 2,3)+\left[\sigma_{; 1 \lambda 23}\right]\left[\sigma_{456}^{; \lambda}\right] .\right.
\end{aligned}
$$

Operating on (C.31) with $\{1-I(3,4)\}$ gives

$$
\begin{aligned}
& \{1+I(5,6)\}\{1-I(3,4)\}\left[\sigma_{; 125346}\right]+\{1-I(3,4)\}\left[\sigma_{; 123456}\right] \\
& \quad+\{1-I(3,4)\}\left[H_{123456}\right] \\
& \quad+\{1+I(5,6)\}\{1-I(3,4)\}(1,5,2,6 ; 3,4) \\
& \quad+\{1+I(5,6)\}\{1-I(3,4)\}(1, \lambda, 2,5)\left[\sigma_{346}^{; \lambda}\right]=0
\end{aligned}
$$

The first term may be evaluated by applying to (C.38) the substitution

$$
\begin{array}{llllll}
1 & 2 & 3 & 4 & 5 & 6 \\
1 & 2 & 5 & 3 & 4 & 6
\end{array}
$$

and it becomes

$$
\begin{aligned}
- & \frac{1}{3}\{1+I(5,6)\}\{1+I(5,6)\}\{1-I(3,4)\}(1,5,2,6 ; 3,4) \\
& -\frac{1}{2}\{1+I(5,6)\}\{1-I(3,4)\}\left((1, \lambda, 2,5)+\left[\sigma_{; 1 \lambda 25}\right]\right)\left[\sigma_{346}^{; \lambda}\right],
\end{aligned}
$$


in which the first term can be simplified, since

$$
\{1+I(5,6)\}\{1+I(5,6)\}=2\{1+I(5,6)\} .
$$

The $[H]$ term in (C.39) is, by (C.29)

$$
\{1+I(5,6)\}\{1-I(3,4)\}\left[\sigma_{; 1 \lambda 25}\right]\left[\sigma_{346}^{; \lambda}\right] .
$$

Thus (C.39) may be written

$$
\begin{gathered}
\{1-I(3,4)\}\left[\sigma_{; 123456}\right]=-\frac{1}{3}\{1+I(5,6)\}\{1-I(3,4)\}(1,5,2,6 ; 3,4) \\
-\frac{1}{2}\{1+I(5,6)\}\{1-I(3,4)\}\left((1, \lambda, 2,5)+\left[\sigma_{; 1 \lambda 25}\right]\right)\left[\sigma_{346}^{; \lambda}\right] .
\end{gathered}
$$

We have now to apply (C.35), (C.38), (C.43) to the solution of (C.31) or (C.32). The first term in (C.32) is

$$
\begin{aligned}
& \{2+(l+I(4,5)+I(5,6) I(4,5)) I(3,4)\}\left[\sigma_{; 123456}\right] \\
& =\{3+(1+I(5,6)) I(4,5)\}\left[\sigma_{; 123456}\right] \\
& \quad-\{1+I(4,5)+I(5,6) I(4,5)\}\{1-I(3,4)\}\left[\sigma_{; 123456}\right]
\end{aligned}
$$

but

$$
\begin{aligned}
& \{3+(1+I(5,6)) I(4,5)\}\left[\sigma_{; 123456}\right] \\
& \quad=\{4+I(5,6)\}\left[\sigma_{; 123456}\right]-\{1+I(5,6)\}\{1-I(4,5)\}\left[\sigma_{; 123456}\right]
\end{aligned}
$$

and

$$
\{4+I(5,6)\}\left[\sigma_{; 123456}\right]=5\left[\sigma_{; 123456}\right]-\{1-I(5,6)\}\left[\sigma_{; 123456}\right]
$$

After adding these three equations, and using (C.35), (C.38), (C.43), we find that the first term of (C.32) is

$$
\begin{aligned}
& 5\left[\sigma_{; 123456}\right]+\frac{1}{3}\{1+I(3,4)\}\{1-I(5,6)\}(1,3,2,4 ; 5,6) \\
& +\frac{1}{3}\{1+I(5,6)\}\{1+I(3,6)\}\{1-I(4,6)\}(1,3,2,6 ; 4,5) \\
& +\frac{1}{2}\{1+I(5,6)\}\{1-I(4,5)\}\left((1, \lambda, 2,3)+\sigma_{; 1 \lambda 23}\right) \sigma_{456}^{; \lambda} \\
& +\frac{1}{3}\{1+I(4,5)+I(5,6) I(4,5)\}\{1+I(5,6)\}\{1-I(3,4)\}(1,5,2,3 ; 6,4) \\
& +\frac{1}{2}\{1+I(4,5)+I(5,6) I(4,5)\}\{1+I(5,6)\}\{1-I(3,4)\} \\
& \cdot\left((1, \lambda, 2,5)+\sigma_{; 1 \lambda 25}\right) \sigma_{346}^{; \lambda} \text {. }
\end{aligned}
$$


Substituting this expression for the first term in (C.32), we get

$$
\begin{aligned}
& 5\left[\sigma_{;} 123456\right] \\
&=-(1,4,2,3 ; 5,6)-(1,5,2,3 ; 4,6)-(1,6,2,3 ; 4,5)-(1, \lambda, 2,3)\left[\sigma_{456}^{; \lambda}\right] \\
&-(1,3,2,4 ; 5,6)-(1,5,2,4 ; 3,6)-(1,6,2,4 ; 3,5)-(1, \lambda, 2,4)\left[\sigma_{356}^{; \lambda}\right] \\
&-(1,3,2,5 ; 4,6)-(1,4,2,5 ; 3,6)-(1,6,2,5 ; 3,4)-(1, \lambda, 2,5)[\sigma ; \lambda 346] \\
&-(1,3,2,6 ; 4,5)-(1,4,2,6 ; 3,5)-(1,5,2,6 ; 3,4)-(1, \lambda, 2,6)\left[\sigma_{345}^{; \lambda}\right] \\
&-\frac{1}{3}((1,3,2,4 ; 5,6)-(1,3,2,4 ; 6,5)+(1,3,2,6 ; 4,5)-(1,3,2,6 ; 5,4) \\
&+(1,4,2,3 ; 5,6)-(1,4,2,3 ; 6,5)+(1,6,2,3 ; 4,5)-(1,6,2,3 ; 5,4) \\
&+(1,3,2,5 ; 4,6)-(1,3,2,5 ; 6,4)+(1,5,2,6 ; 3,4)-(1,5,2,6 ; 4,3) \\
&+(1,5,2,3 ; 4,6)-(1,5,2,3 ; 6,4)+(1,6,2,5 ; 3,4)-(1,6,2,5 ; 4,3) \\
&+(1,4,2,6 ; 3,5)-(1,4,2,6 ; 5,3)+(1,4,2,5 ; 3,6)-(1,4,2,5 ; 6,3) \\
&+(1,6,2,4 ; 3,5)-(1,6,2,4 ; 5,3)+(1,5,2,4 ; 3,6)-(1,5,2,4 ; 6,3)) \\
&-\frac{1}{2}\left((1, \lambda, 2,3)+\left[\sigma_{; 1 \lambda 23}\right]\right)\left(\left[\sigma_{456}^{; \lambda}\right]-\left[\sigma_{546}^{; \lambda}\right]+\left[\sigma_{465}^{; \lambda}\right]-\left[\sigma_{645}^{; \lambda}\right]\right) \\
&-\frac{1}{2}\left((1, \lambda, 2,4)+\left[\sigma_{; 1 \lambda 24}\right]\right)\left(\left[\sigma_{356}^{; \lambda}\right]-\left[\sigma_{536}^{; \lambda}\right]+\left[\sigma_{365}^{; \lambda}\right]-\left[\sigma_{356}^{; \lambda}\right]\right) \\
&-\frac{1}{2}\left((1, \lambda, 2,5)+\left[\sigma_{; 1 \lambda 25}\right]\right)\left(\left[\sigma_{346}^{; \lambda}\right]-\left[\sigma_{436}^{; \lambda}\right]+\left[\sigma_{364}^{; \lambda}\right]-\left[\sigma_{634}^{; \lambda}\right]\right) \\
&-\frac{1}{2}\left((1, \lambda, 2,6)+\left[\sigma_{; 1 \lambda 26}\right]\right)\left(\left[\sigma_{345}^{; \lambda}\right]-\left[\sigma_{435}^{; \lambda}\right]+\left[\sigma_{354}^{; \lambda}\right]-\left[\sigma_{534}^{; \lambda}\right]\right) \\
&-[H 123456] .
\end{aligned}
$$

The last term in the first line, the terms in the eleventh line, and the first term in $-\left[H_{123456}\right]$ (see Eq. (C.29)), together make up (by use (C.18) and (C.20))

$$
\begin{aligned}
\{(1, & \left.\lambda, 2,3)+\left[\sigma_{; 1 \lambda 23}\right]\right\}\left\{-2\left[\sigma_{456}^{; \lambda}\right]+\frac{1}{2}\left(\left[\sigma_{546}^{; \lambda}\right]+\left[\sigma_{645}^{; \lambda}\right]\right)\right. \\
= & \left\{-(2,3, \lambda, 1)-\frac{1}{3}((1,2, \lambda, 3)+(1,3, \lambda, 2))\right\} \\
& \cdot\left\{\frac{2}{3} g^{\lambda \mu}((\mu, 5,4,6)+(\mu, 6,4,5))-\frac{1}{6} g^{\lambda \mu}((\mu, 6,5,4)+(\mu, 5,6,4))\right\} \\
= & -\frac{5}{6} g^{\lambda \mu}\left\{(2,3, \lambda, 1)+\frac{1}{3}(1,2, \lambda, 3)+\frac{1}{3}(1,3, \lambda, 2)\right\} \\
& \cdot\{(\mu, 5,4,6)+(\mu, 6,4,5)\} \\
= & -\frac{5}{6} g^{\lambda \mu}\left\{(2,3, \lambda, 1)+\frac{1}{3}(1,2, \lambda, 3)+\frac{1}{3}(1,3, \lambda, 2)\right\} \\
& \cdot\{(4,5, \mu, 6)+(4,6, \mu, 5)\} .
\end{aligned}
$$

But

$$
(2,3, \lambda, 1)+(2, \lambda, 1,3)+(2,1,3, \lambda)=0
$$

and therefore

$$
(2,3, \lambda, 1)=-(2, \lambda, 1,3)-(2,1,3, \lambda),
$$

so that (C.48) is equal to

$$
-\frac{5}{9} g^{\lambda \mu}\{2(1,3, \lambda, 2)-(1,2, \lambda, 3)\}\{(4,5, \mu, 6)+(4,6, \mu, 5)\} .
$$

The first of the remaining terms in $-\left[H_{123456}\right]$ which are not used in forming the previous expression and the three expressions similar to it, is (see (C.29)) equal to

$$
-\frac{1}{9} g^{\lambda \mu}\{(1,3, \lambda, 4)+(1,4, \lambda, 3)\}\{(2,3, \mu, 6)+(2,6, \mu, 3)\} .
$$


Thus we see that (C.47) may be written

$$
\begin{aligned}
{\left[\sigma_{;}\right.} & \\
= & -\frac{4}{15}\{(1,36] \\
& +(1,4,2,3 ; 5,6)+(1,4,2,5 ; 3,6)+(1,4,2,6 ; 3,5) \\
& +(1,5,2,3 ; 4,6)+(1,5,2,4 ; 3,6)+(1,5,2,6 ; 3,4) \\
& +(1,6,2,3 ; 4,5)+(1,6,2,4 ; 3,5)+(1,6,2,5 ; 3,4)\} \\
& -\frac{1}{15}\{(1,3,2,4 ; 6,5)+(1,3,2,5 ; 6,4)+(1,3,2,6 ; 5,4) \\
& +(1,4,2,3 ; 6,5)+(1,4,2,5 ; 6,3)+(1,4,2,6 ; 5,3) \\
& +(1,5,2,3 ; 6,4)+(1,5,2,4 ; 6,3)+(1,5,2,6 ; 4,3) \\
& +(1,6,2,3 ; 5,4)+(1,6,2,4 ; 5,3)+(1,6,2,5 ; 4,3)\} \\
& -\frac{1}{9} g^{\lambda \mu} \sum_{i=1}^{4} A_{\lambda \mu 123456}^{(i)} \\
& -\frac{1}{45} g^{\lambda \mu} \sum_{j=1}^{6} B_{\lambda \mu 123456}^{(i)}
\end{aligned}
$$

where

$$
\begin{aligned}
& A_{\lambda \mu 123456}^{(1)}=(2(1,3, \lambda, 2)-(1,2, \lambda, 3))((4,5, \mu, 6)+(4,6, \mu, 5)), \\
& A_{\lambda \mu 123456}^{(2)}=(2(1,4, \lambda, 2)-(1,2, \lambda, 4))((3,5, \mu, 6)+(3,6, \mu, 5)), \\
& A_{\lambda \mu 123456}^{(3)}=(2(1,5, \lambda, 2)-(1,2, \lambda, 5))((3,4, \mu, 6)+(3,6, \mu, 4)), \\
& A_{\lambda \mu 123456}^{(4)}=(2(1,6, \lambda, 2)-(1,2, \lambda, 6))((3,4, \mu, 5)+(3,5, \mu, 4)), \\
& B_{\lambda \mu 123456}^{(1)}=((1,3, \lambda, 4)+(1,4, \lambda, 3))((2,5, \mu, 6)+(2,6, \mu, 5)), \\
& B_{\lambda \mu 123456}^{(2)}=((1,3, \lambda, 5)+(1,5, \lambda, 3))((2,4, \mu, 6)+(2,6, \mu, 4)), \\
& B_{\lambda \mu 123456}^{(3)}=((1,3, \lambda, 6)+(1,6, \lambda, 3))((2,4, \mu, 5)+(2,5, \mu, 4)), \\
& B_{\lambda \mu 123456}^{(4)}=((1,4, \lambda, 5)+(1,5, \lambda, 4))((2,3, \mu, 6)+(2,6, \mu, 3)), \\
& B_{\lambda \mu 123456}^{(5)}=((1,4, \lambda, 6)+(1,6, \lambda, 4))((2,3, \mu, 5)+(2,5, \mu, 3)), \\
& B_{\lambda \mu 123456}^{(6)}=((1,5, \lambda, 6)+(1,6, \lambda, 5))((2,3, \mu, 4)+(2,4, \mu, 3)) .
\end{aligned}
$$

\section{Coefficient bivectors: coincidence limits}

We will derive the coincidence limits of the following coefficient bivectors: [ $\left.b_{1 \mu v^{\prime}}\right],\left[b_{1 \mu v^{\prime} ; \rho}\right]$, $\left[b_{1 \mu v^{\prime} ; \rho \omega}\right],\left[b_{2 \mu v^{\prime}}\right]$; these quantities, together with the fundamental formula for the derivatives of the coincidence limit of bitensors

$$
\left[T_{\alpha_{1} \alpha_{2} \ldots \beta_{1}^{\prime} \beta_{2}^{\prime} \ldots}\left(x, x^{\prime}\right)\right]_{; \gamma}=\left[T_{\alpha_{1} \alpha_{2} \ldots \beta_{1}^{\prime} \beta_{2}^{\prime} \ldots ; \gamma^{\prime}}\left(x, x^{\prime}\right)\right]+\left[T_{\alpha_{1} \alpha_{2} \ldots \beta_{1}^{\prime} \beta_{2}^{\prime} \ldots ; \gamma}\left(x, x^{\prime}\right)\right]
$$

make it possible to evaluate explicitly the right-hand side in (3.19). 
D.1 Coincidence limit of $b_{1 \mu v^{\prime}}$

Consider the recurrence relation (2.21) for the coefficient bivectors $b_{n \mu v^{\prime}}$ :

$$
\sigma^{; \lambda} b_{n \mu \nu^{\prime} ; \lambda}+n b_{n \mu \nu^{\prime}}=\frac{1}{\sqrt{\Delta}}\left(\sqrt{\Delta} b_{n-1 \mu v^{\prime}}\right) ; \lambda{ }^{\lambda}-R_{\mu}^{\lambda} b_{n-1} \lambda v^{\prime} .
$$

Since $b_{0 \mu v^{\prime}}=g_{\mu v^{\prime}}$, for $n=1$ the previous equation is

$$
\begin{aligned}
& \sigma^{; \lambda} b_{1 \mu v^{\prime} ; \lambda}+b_{1 \mu v^{\prime}}=\frac{1}{\sqrt{\Delta}}\left(\sqrt{\Delta} g_{\mu v^{\prime}}\right) ; \lambda \\
& \quad=\frac{1}{\sqrt{\Delta}}\left(\sqrt{\Delta}_{; \lambda}^{\lambda} g_{\mu v^{\prime}}+2 \sqrt{\Delta}_{\mu \lambda}^{\lambda} g_{\mu v^{\prime} ;}{ }^{\lambda}+\sqrt{\Delta} g_{\mu v^{\prime} ; \lambda}{ }^{\lambda}\right)-R_{\mu}^{\lambda} g_{\lambda v^{\prime}} .
\end{aligned}
$$

Taking the coincidence limit, and using (see Ref. [33])

$$
\begin{aligned}
{[\sigma ; \lambda] } & =0, \\
{[\sqrt{\Delta}] } & =1, \\
{\left[\sqrt{\Delta}_{\mu}\right] } & =0, \\
{\left[\sqrt{\Delta}_{; \mu \nu}\right] } & =\frac{1}{6} R_{\mu \nu}, \\
{\left[g_{\mu v^{\prime}}\right] } & =g_{\mu \nu}, \\
{\left[g_{\mu v^{\prime} ; \alpha}\right] } & =0, \\
{\left[g_{\mu v^{\prime} ; \alpha \beta}\right] } & =-\frac{1}{2} R_{\mu \nu \alpha \beta},
\end{aligned}
$$

one obtains

$$
\left[b_{1 \mu v^{\prime}}\right]=\frac{1}{6} R g_{\mu \nu}-R_{\mu \nu} .
$$

D. 2 Coincidence limit of $b_{1 \mu v^{\prime} ; \rho}$

Taking the first derivative of (D.2), one obtains

$$
\begin{aligned}
\sigma_{\rho}^{\lambda} & b_{1 \mu v^{\prime} ; \lambda}+\sigma^{\lambda} b_{1 \mu v^{\prime} ; \lambda \rho}+b_{1 \mu v^{\prime} ; \rho} \\
= & \left(\frac{1}{\sqrt{\Delta}}\right)_{; \rho}\left(\sqrt{\Delta}_{; \lambda}^{\lambda} g_{\mu v^{\prime}}+2 \sqrt{\Delta}_{; \lambda} g_{\mu v^{\prime} ;}^{\lambda}+\sqrt{\Delta} g_{\mu v^{\prime} ; \lambda}{ }^{\lambda}\right) \\
& +\frac{1}{\sqrt{\Delta}}\left(\sqrt{\Delta}_{; \lambda}^{\lambda} g_{\mu v^{\prime}}+2 \sqrt{\Delta_{; \lambda}} g_{\mu v^{\prime} ;}^{\lambda}+\sqrt{\Delta} g_{\mu v^{\prime} ; \lambda}{ }^{\lambda}\right)_{; \rho} \\
& -R_{\mu ; \rho}^{\lambda} g_{\lambda v^{\prime}}-R_{\mu}^{\lambda} g_{\lambda v^{\prime} ; \rho} .
\end{aligned}
$$

Taking the coincidence limit, and using (D.3)-(D.9) and

$$
\begin{aligned}
{\left[\left(\frac{1}{\sqrt{\Delta}}\right)_{; \rho}\right] } & =-\left[(\sqrt{\Delta})^{-2}\right]\left[\sqrt{\Delta}_{; \rho}\right]=0, \\
{\left[\sigma_{; \mu \nu}\right] } & =g_{\mu \nu},
\end{aligned}
$$

one obtains

$$
2\left[b_{1 \mu v^{\prime} ; \rho}\right]=\left[{\sqrt{\Delta_{; \lambda}}}^{\lambda} \rho\right] g_{\mu \nu}+\left[g_{\mu v^{\prime} ; \lambda}{ }^{\lambda} \rho\right]-R_{\mu \nu ; \rho} .
$$


From

$$
\begin{aligned}
& {\left[{\sqrt{\Delta_{;}}}_{\alpha \beta \gamma}\right]=\frac{1}{12}\left(R_{\alpha \beta ; \gamma}+R_{\alpha \gamma ; \beta}+R_{\beta \gamma ; \alpha}\right),} \\
& {\left[g_{\mu \nu^{\prime} ; \alpha \beta \gamma}\right]=-\frac{1}{3}\left(R_{\mu \nu \alpha \beta ; \gamma}+R_{\mu \nu \alpha \gamma ; \beta}\right),}
\end{aligned}
$$

one arrives at

$$
\begin{aligned}
& {\left[{\sqrt{\Delta_{; \lambda}}}^{\lambda} \rho\right]=\frac{1}{12}\left(R_{; \gamma}+2 R_{\lambda \rho ;}{ }^{\lambda}\right),} \\
& {\left[g_{\mu \nu^{\prime} ; \lambda}{ }^{\lambda}\right]=-\frac{1}{3} R_{\mu \nu \alpha \gamma} ; \alpha .}
\end{aligned}
$$

Then (D.14) reads

$$
\left[b_{1 \mu \nu^{\prime} ; \rho}\right]=\frac{1}{24} g_{\mu \nu}\left(R_{; \gamma}+2 R_{\lambda \rho ;}{ }^{\lambda}\right)-\frac{1}{6} R_{\mu \nu \alpha \gamma} ; \alpha-\frac{1}{2} R_{\mu \nu ; \rho} .
$$

D.3 Coincidence limit of $b_{1 \mu \nu^{\prime} ; \rho \omega}$

Upon differentiating again (D.2), one obtains

$$
\begin{aligned}
& \sigma_{;}^{\lambda} \rho \omega b_{1 \mu v^{\prime} ; \lambda}+\sigma_{; \rho}^{\lambda} b_{1 \mu v^{\prime} ; \lambda \omega}+\sigma_{;}^{\lambda} b_{1 \mu v^{\prime} ; \lambda \rho}+\sigma_{;}^{\lambda} b_{\mu v^{\prime} ; \lambda \rho \omega}+b_{1 \mu v^{\prime} ; \rho \omega} \\
& =\left(\frac{1}{\sqrt{\Delta}}\right)_{; \rho \omega}\left(\sqrt{\Delta}_{; \lambda}^{\lambda} g_{\mu \nu^{\prime}}+2 \sqrt{\Delta_{; \lambda}} g_{\mu v^{\prime} ;}^{\lambda}+\sqrt{\Delta} g_{\mu v^{\prime} ; \lambda} \lambda^{\lambda}\right) \\
& +\left(\frac{1}{\sqrt{\Delta}}\right)_{; \rho}\left(\sqrt{\Delta}_{; \lambda}^{\lambda} g_{\mu v^{\prime}}+2 \sqrt{\Delta_{; \lambda}} g_{\mu \nu^{\prime} ;}^{\lambda}+\sqrt{\Delta} g_{\mu v^{\prime} ; \lambda}{ }^{\lambda}\right)_{; \omega} \\
& +\left(\frac{1}{\sqrt{\Delta}}\right)_{; \omega}\left(\sqrt{\Delta}_{; \lambda}^{\lambda} g_{\mu \nu^{\prime}}+2 \sqrt{\Delta_{; \lambda}} g_{\mu \nu^{\prime} ;}^{\lambda}+\sqrt{\Delta} g_{\mu v^{\prime} ; \lambda}{ }^{\lambda}\right)_{; \rho} \\
& +\left(\frac{1}{\sqrt{\Delta}}\right)\left(\sqrt{\Delta}_{; \lambda}^{\lambda} g_{\mu v^{\prime}}+2 \sqrt{\Delta}_{; \lambda} g_{\mu v^{\prime} ;}^{\lambda}+\sqrt{\Delta} g_{\mu \nu^{\prime} ; \lambda}{ }^{\lambda}\right)_{; \rho \omega} \\
& -R_{\mu}^{\lambda ; \rho \omega} g_{\lambda v^{\prime}}-R_{\mu}^{\lambda}{ }^{\lambda} g_{\lambda v^{\prime} ; \omega}-R_{\mu}^{\lambda ; \omega} g_{\lambda v^{\prime} ; \rho}-R_{\mu}{ }^{\lambda} g_{\lambda v^{\prime} ; \rho \omega}
\end{aligned}
$$

Taking the coincidence limit, and using

$$
\begin{aligned}
& {\left[\sigma_{; \alpha \beta \gamma}\right]=0} \\
& {\left[\Delta_{; \alpha \beta}^{-1 / 2}\right]=\left[\left(-\Delta_{; \alpha \beta}^{1 / 2} / \Delta\right)+\left(2 \Delta_{; \alpha}^{1 / 2} \Delta_{; \beta}^{1 / 2} / \Delta^{3 / 2}\right)\right]=-\frac{1}{6} R_{\alpha \beta},}
\end{aligned}
$$

one arrives at

$$
\begin{aligned}
& 2\left[b_{1 \mu v^{\prime} ; \rho \omega}\right]+\left[b_{1 \mu v^{\prime} ; \omega \rho}\right] \\
& =-\frac{1}{36} R g_{\mu \nu} R_{\rho \omega}+\left[\begin{array}{cc}
\Delta^{1 / 2} & \lambda \\
; \lambda & \rho \omega
\end{array}\right] g_{\mu \nu}-\frac{1}{12} R R_{\mu \nu \rho \omega} \\
& -\frac{1}{6} R_{\rho}^{\lambda} R_{\mu \nu \lambda \omega}-\frac{1}{6} R_{\omega}^{\lambda} R_{\mu \nu \lambda \rho}+\left[g_{\mu \nu^{\prime} ; \lambda}{ }^{\lambda} \rho \omega\right] \\
& -R_{\mu \nu ; \rho \omega}+\frac{1}{2} R_{\mu}{ }^{\lambda} R_{\lambda \nu \rho \omega} .
\end{aligned}
$$

Now we can exploit the close relation between the commutator of covariant derivatives acting on a (co-)vector and the Riemann tensor:

$$
b_{1 \mu \nu^{\prime} ; \rho \omega}-b_{1 \mu v^{\prime} ; \omega \rho}=-R_{\mu}{ }^{\sigma} \rho \omega b_{1 \sigma \nu^{\prime}}
$$

hence, on taking the limit, we have

$$
\left[b_{1 \mu v^{\prime} ; \rho \omega}\right]-\left[b_{1 \mu v^{\prime} ; \omega \rho}\right]=-R_{\mu}{ }^{\sigma} \rho \omega\left[b_{1 \sigma v^{\prime}}\right] .
$$


Inserting this equation in (D.21), one obtains

$$
\begin{aligned}
& 3\left[b_{1 \mu \nu^{\prime} ; \rho \omega}\right]=-R_{\mu}{ }^{\sigma} \rho \omega\left[b_{1 \sigma v^{\prime}}\right]
\end{aligned}
$$

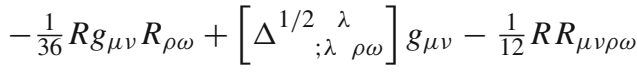

$$
\begin{aligned}
& -\frac{1}{6} R_{\rho}^{\lambda} R_{\mu \nu \lambda \omega}-\frac{1}{6} R_{\omega}^{\lambda} R_{\mu \nu \lambda \rho}+\left[g_{\mu \nu^{\prime} ; \lambda}{ }^{\lambda} \rho \omega\right] \\
& -R_{\mu \nu ; \rho \omega}+\frac{1}{2} R_{\mu}{ }^{\lambda} R_{\lambda \nu \rho \omega},
\end{aligned}
$$

which can be fully expanded using

$$
\begin{aligned}
& S_{\lambda \mu \nu \rho} \equiv-\frac{1}{3}\left(R_{\lambda \nu \mu \rho}+R_{\lambda \rho \mu \nu}\right), \\
& {\left[\sqrt{\Delta}_{; \alpha \beta \gamma \delta}\right]=-\frac{1}{8}\left\{\left[\sigma_{\rho \alpha \beta \gamma \delta}^{; \rho}\right]\right.} \\
& -\frac{1}{3}\left(R_{\alpha \rho} R_{\beta \gamma \delta}^{\rho}+R_{\beta \rho} R_{\alpha \gamma \delta}^{\rho}+R_{\gamma \rho} R_{\alpha \beta \delta}^{\rho}+R_{\delta \rho} R_{\alpha \beta \gamma}^{\rho}\right) \\
& +\frac{1}{3}\left(R_{\alpha \rho} S_{\beta \gamma \delta}^{\rho}+R_{\beta \rho} S_{\alpha \gamma \delta}^{\rho}+R_{\gamma \rho} S_{\alpha \beta \delta}^{\rho}+R_{\delta \rho} S_{\alpha \beta \gamma}^{\rho}\right) \\
& \left.-\frac{2}{9}\left(R_{\alpha \beta} R_{\gamma \delta}+R_{\alpha \gamma} R_{\beta \delta}+R_{\alpha \delta} R_{\beta \gamma}\right)\right\} \text {, } \\
& {\left[g_{\alpha \beta^{\prime} ; \mu \nu \sigma \tau}\right]=-\frac{1}{4}\left(R_{\alpha \beta \mu \nu ; \sigma \tau}+R_{\alpha \beta \mu \sigma ; \nu \tau}+R_{\alpha \beta \mu \tau ; \nu \sigma}\right)} \\
& +\frac{1}{8}\left(R_{\alpha \beta \rho \tau} S_{\mu \nu \sigma}^{\rho}+R_{\alpha \beta \rho \sigma} S_{\mu \nu \tau}^{\rho}+R_{\alpha \beta \rho \nu} S_{\mu \sigma \tau}^{\rho}+\right. \\
& \left.+R_{\alpha \beta \rho \mu} S_{\nu \sigma \tau}^{\rho}\right) \\
& -\frac{1}{8}\left(R_{\beta \sigma \tau}^{\rho} R_{\rho \alpha \mu \nu}+R_{\beta \mu \nu}^{\rho} R_{\rho \alpha \sigma \tau}+R_{\alpha \beta \rho \tau} R_{\mu \nu \sigma}^{\rho}\right. \\
& +R_{\alpha \beta \rho \sigma} R_{\mu \nu \tau}^{\rho}+R_{\beta \mu \tau}^{\rho} R_{\rho \alpha \nu \sigma}+R_{\beta \nu \sigma}^{\rho} R_{\rho \alpha \mu \tau} \\
& +R_{\beta \nu \tau}^{\rho} R_{\rho \alpha \mu \sigma}+R_{\beta \mu \sigma}^{\rho} R_{\rho \alpha \nu \tau}+R_{\alpha \beta \rho \nu} R_{\mu \sigma \tau}^{\rho} \\
& \left.+R_{\alpha \beta \mu \rho} R_{\nu \sigma \tau}^{\rho}\right) \text {, }
\end{aligned}
$$

D.4 Coincidence limit of $b_{2} \mu v^{\prime}$

For $n=2$, Eq. (2.21) is:

$$
\begin{aligned}
& \sigma^{; \lambda} b_{2 \mu v^{\prime} ; \lambda}+2 b_{2} \mu v^{\prime} \\
& \quad=\frac{1}{\sqrt{\Delta}}\left(\sqrt{\Delta}_{; \lambda}^{\lambda} b_{1 \mu v^{\prime}}+2{\sqrt{\Delta_{;}}}_{; \lambda} b_{1 \mu v^{\prime} ;}{ }^{\lambda}+\sqrt{\Delta} b_{1 \mu v^{\prime} ; \lambda}{ }^{\lambda}\right)-R_{\mu}^{\lambda} b_{1 \lambda v^{\prime}}
\end{aligned}
$$

Taking the coincidence limit, one obtains

$$
\begin{aligned}
& 2\left[b_{2 \mu v^{\prime}}\right]=\frac{1}{6} R\left[b_{1 \mu v^{\prime}}\right]+\left[b_{1 \mu v^{\prime}}\right]_{; \lambda}^{\lambda}-R_{\mu}^{\lambda}\left[b_{1 \lambda v^{\prime}}\right] \\
& =\left(\frac{1}{36} R^{2} g_{\mu \nu}-\frac{1}{6} R R_{\mu \nu}\right)+\left\{\frac{1}{3} g_{\mu \nu}\left(-\frac{1}{36} R^{2}+\left[\begin{array}{ccc}
\sqrt{\Delta}^{1 / 2} & \alpha \beta & \beta \\
\alpha & \beta
\end{array}\right]\right)\right. \\
& \left.-\frac{1}{3} \square R_{\mu \nu}+\frac{1}{3}\left[g_{\mu \nu^{\prime} ; \alpha \beta} \alpha^{\beta}\right]\right\}+\left(-\frac{1}{6} R R_{\mu \nu}+R_{\mu}^{\lambda} R_{\lambda \nu}\right) .
\end{aligned}
$$

By using (D.26), (D.27), one arrives at

$$
\begin{aligned}
{\left[\begin{array}{ccc}
\sqrt{\Delta}^{1 / 2} & \alpha & \beta \\
; \alpha & \beta
\end{array}\right] } & =\frac{1}{5} \square R+\frac{1}{36} R^{2}-\frac{1}{30} R_{\alpha \beta} R^{\alpha \beta}+\frac{1}{30} R_{\alpha \beta \gamma \delta} R^{\alpha \beta \gamma \delta}, \\
{\left[\begin{array}{ccc}
g_{\mu \nu^{\prime} ; \alpha} & \beta & \beta
\end{array}\right] } & =-\frac{1}{2} R_{\mu}^{\alpha \beta \gamma}{ }_{\mu} R_{\alpha \beta \gamma \nu} .
\end{aligned}
$$


Hence (D.29) reads

$$
\begin{aligned}
2\left[b_{2 \mu \nu^{\prime}}\right]= & R_{\mu}^{\lambda} R_{\lambda \nu}-\frac{1}{3} R R_{\mu \nu}-\frac{1}{3} \square R_{\mu \nu}-\frac{1}{6} R_{\mu}^{\alpha \beta \gamma} R_{\alpha \beta \gamma \nu} \\
& +\left(\frac{1}{36} R^{2}+\frac{1}{15} \square R-\frac{1}{90} R_{\alpha \beta} R^{\alpha \beta}+\frac{1}{90} R^{\alpha \beta \gamma \delta} R_{\alpha \beta \gamma \delta}\right) g_{\mu \nu} .
\end{aligned}
$$

\section{References}

1. B.S. DeWitt, The Global Approach to Quantum Field Theory (Oxford University Press, Oxford, 2003)

2. S.W. Hawking, Black hole explosions? Nature 248, 30-31 (1974)

3. S.W. Hawking, Particle creation by black holes. Commun. Math. Phys. 43, 199-220 (1975)

4. W.G. Unruh, Notes on black-hole evaporation. Phys. Rev. D 14, 870-892 (1976)

5. N.D. Birrell, P.C.W. Davies, Quantum Fields in Curved Space (Cambridge University Press, Cambridge, 1982)

6. S.A. Fulling, Aspects of Quantum Field Theory in Curved Spacetime (Cambridge University Press, Cambridge, 1989)

7. L. Parker, D. Toms, Quantum Field Theory in Curved Spacetime: Quantized Fields and Gravity (Cambridge University Press, Cambridge, 2009)

8. B.S. DeWitt, Dynamical Theory of Groups and Fields (Gordon \& Breach, New York, 1965)

9. B.S. DeWitt, Quantum field theory in curved spacetime. Phys. Rep. 19, 295-357 (1975)

10. B.S. DeWitt, The spacetime approach to quantum field theory, in Relativity, Groups and Topology II, ed. by B.S. DeWitt, R. Stora (North-Holland, Amsterdam, 1984), pp. 381-738

11. B. Simon, Functional Integration and Quantum Physics (Academic Press, New York, 1979)

12. J. Glimm, A. Jaffe, Quantum Physics: A Functional Integral Point of View (Springer, Berlin, 2012)

13. P. Cartier, C. DeWitt-Morette, Functional Integration: Action and Symmetries (Cambridge University Press, Cambridge, 2006)

14. S.M. Christensen, Regularization, renormalization, and covariant geodesic point separation. Phys. Rev. D 17, 946-963 (1978)

15. G. Bimonte, E. Calloni, L. Di Fiore, G. Esposito, L. Milano, L. Rosa, On the photon Green functions in curved spacetime. Class. Quantum Gravity 21, 647-659 (2004)

16. A. Heck, FORM for Pedestrians. https://www.nikhef.nl/ form/ (2000)

17. J.A.M. Vermaseren, T. Kaneko, J. Kuipers, B. Ruijl, M. Tentyukov, T. Ueda, J. Vollinga, FORM Version 4.2 Reference Manual. https://www.nikhef.nl/ form/ (2018)

18. Courses, https://www.nikhef.nl/ form/

19. S.A. Teukolsky, The Kerr metric. Class. Quantum Gravity 32, 124006 (2015)

20. M. Realdi, G. Peruzzi, Einstein, de Sitter and the beginning of relativistic cosmology in 1917. Gen. Relativ. Gravit. 41, 225-247 (2009)

21. R. Endo, Gauge dependence of the gravitational conformal anomaly for the electromagnetic field. Prog. Theor. Phys. 71, 1366-1384 (1984)

22. V. Fock, Proper-time in classical and quantum mechanics. Phys. Z. Sowjetunion 12, 404-425 (1937)

23. J. Schwinger, On gauge invariance and vacuum polarization. Phys. Rev. 82, 664-679 (1951)

24. B.L. Giacchini, T. de Paula Netto, I.L. Shapiro, On the Vilkovisky-DeWitt approach and renormalization group in effective quantum gravity. JHEP 10, 011 (2020)

25. M.R. Brown, A.C. Ottewill, Photon propagators and the definition and approximation of renormalized stress tensors in curved space-time. Phys. Rev. D 34, 1776-1786 (1986)

26. B.P. Jensen, A. Ottewill, Renormalized electromagnetic stress tensor in Schwarzschild spacetime. Phys. Rev. D 39, 1130-1138 (1989)

27. S.M. Christensen, Ph.D. dissertation (University of Texas at Austin, 1975, unpublished)

28. M.J. Duff, Observations on conformal anomalies. Nucl. Phys. B 125, 334-348 (1977)

29. S.M. Christensen, M.J. Duff, Axial and conformal anomalies for arbitrary spin in gravity and supergravity. Phys. Lett. B 76, 571-574 (1978)

30. B.S. Kay, Quantum field theory in curved spacetime. arXiv:gr-qc/0601008v2 (2006)

31. R.E. Peierls, The commutation laws of relativistic field theory. Proc. R. Soc. Lond. A 214, 143-152 (1952)

32. J.L. Synge, A characteristic function in Riemannian space and its application to the solution of geodesic triangles. Proc. Lond. Math. Soc. 2, 241-258 (1931)

33. J.L. Synge, Relativity: The General Theory (North-Holland, Amsterdam, 1960) 